\title{
The Neurosurgical Thneed: Expected and Unexpected Neurosurgical Misadventures of the Brain and Spine
}

J Sanders MD, CY Chung MD PhD, JJ O'Keefe MD, N Kinger MD, B Weinberg MD PhD, ME Zygmont MD, RB Peterson MD

Department of Radiology and Imaging Sciences, Emory University School of Medicine, Atlanta, GA American Society of Neuroradiology, Annual Meeting 2020

Presentation Number: 2120

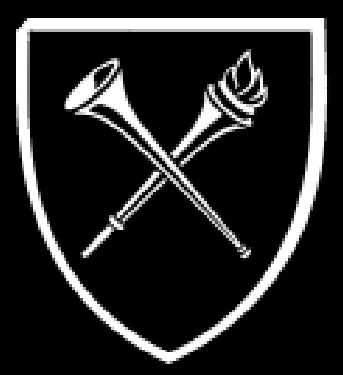

EMORY

UNIVERSITY

$S \mathrm{CHOOL} O \mathrm{O}$

MEDICINE 


\section{Vascular and Retraction Related Injuries}


Case 1 


\section{CASE 1: Occipital Anaplastic Astrocytoma Resection}

- History: 35-year-old male with biopsy proven left occipital anaplastic astrocytoma, progressing despite radiation and Temozolomide with Avastin

- Pre-op Imaging findings: Left anteromedial occipital rim enhancing mass abutting the tentorium, demonstrating internal $\mathrm{T} 2$ hyperintense signal

- Surgery: Mass resection via suboccipital craniotomy

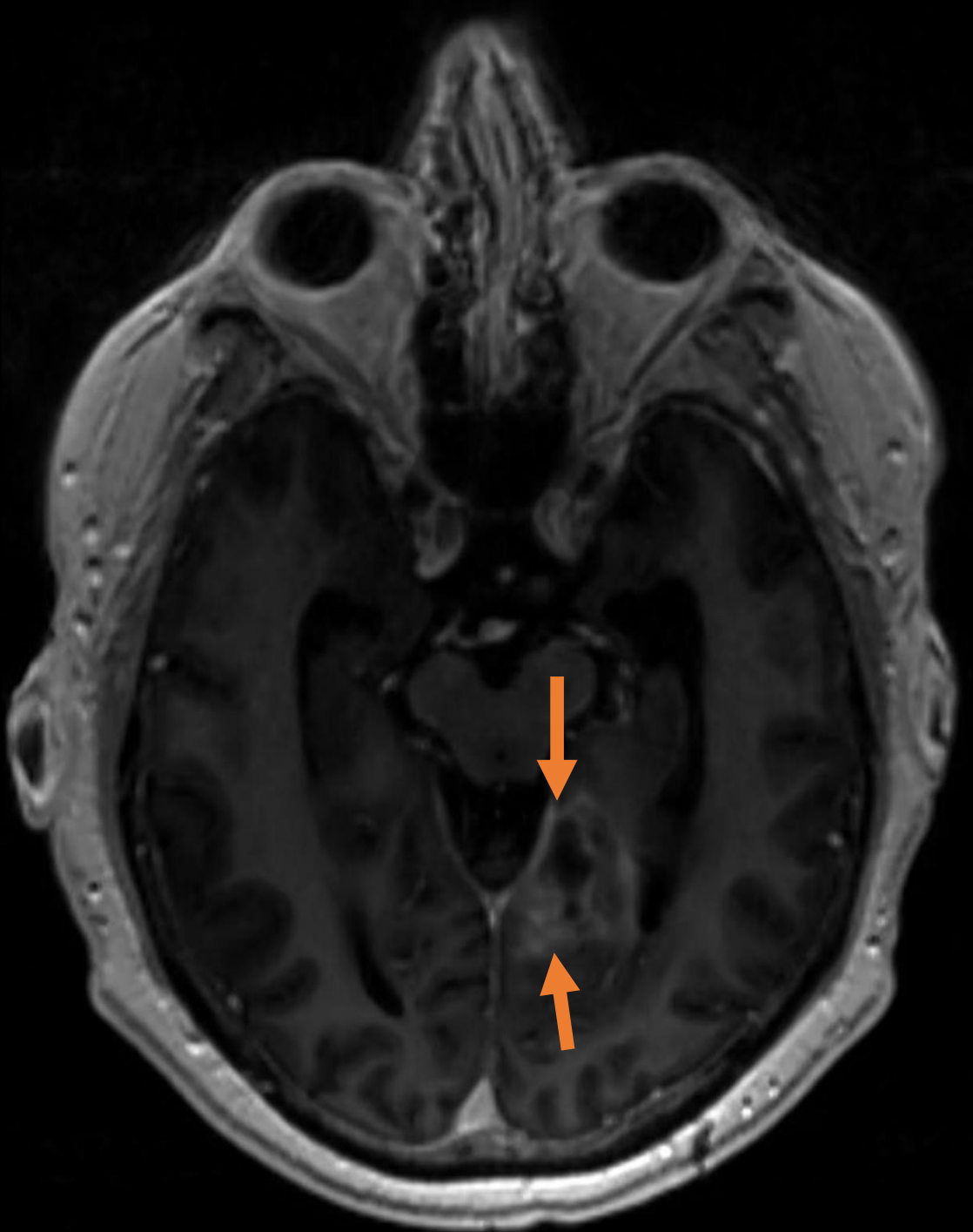

Ax T1 Post

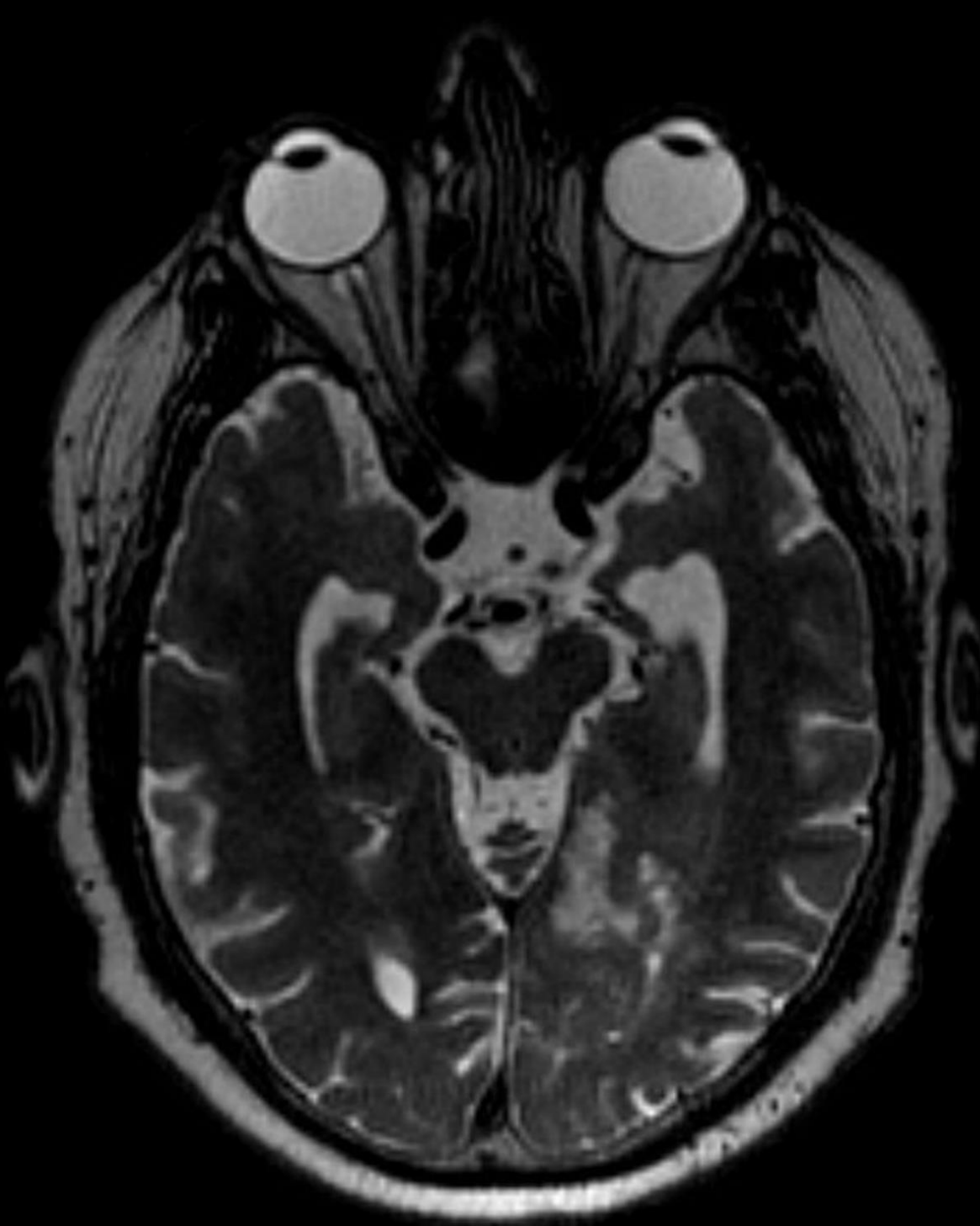

Ax T2 


\section{Postoperative Unexpected Finding: Persistent Headache and Right Visual Field Cut}

T1 Pre (above)

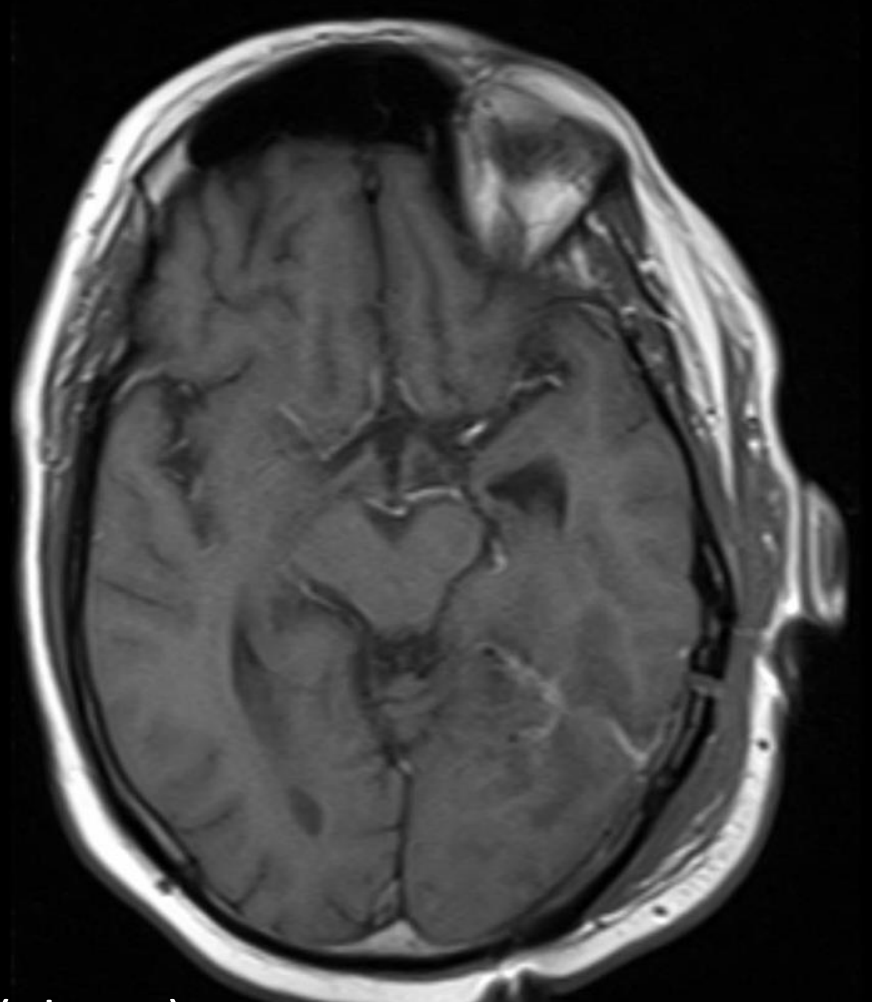

T1 Post (below)

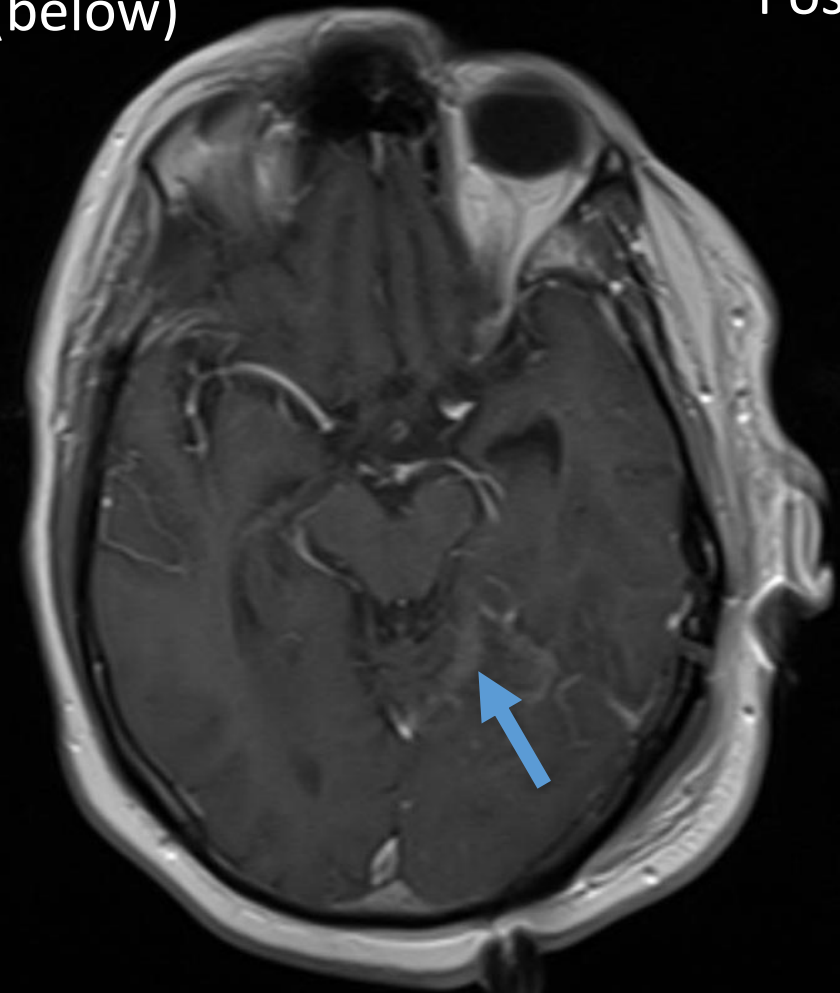

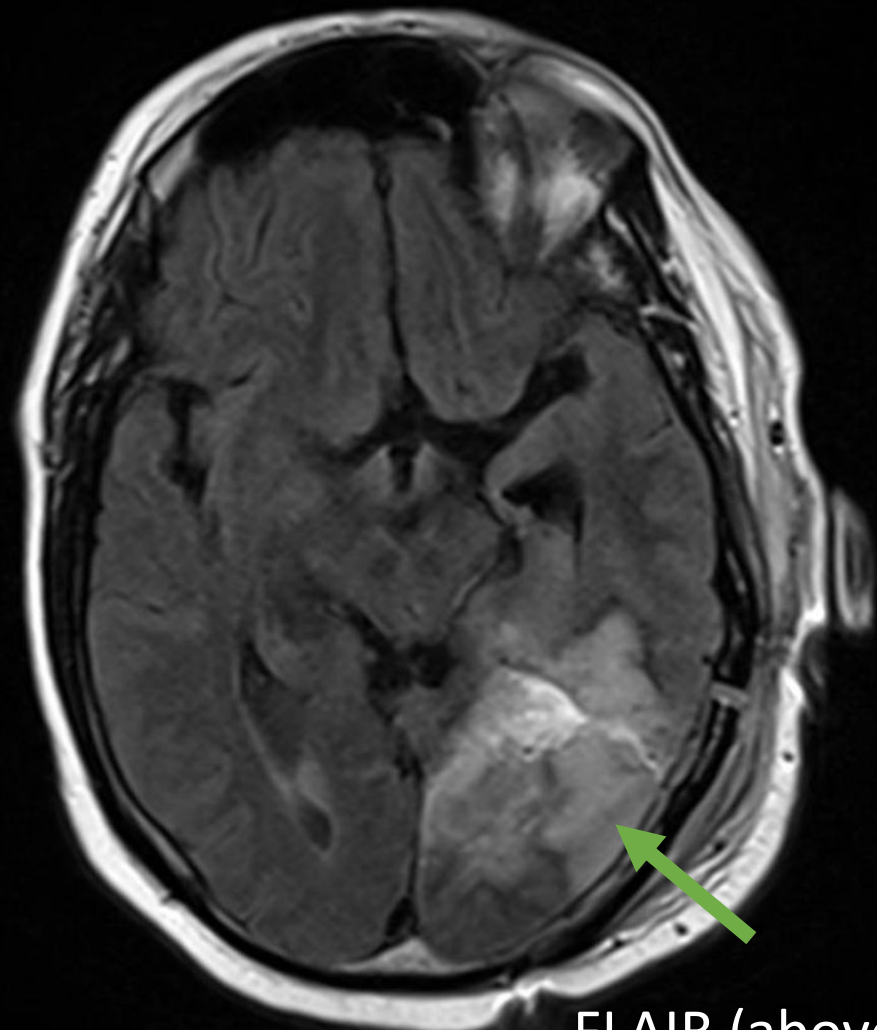

FLAIR (above) DWI (below)

- Enhancement along the resection cavity favored to represent expected early granulation tissue

- Unexpected finding: Large territory of diffusion restriction concerning for territorial infarct surrounding the resection cavity with corresponding edema

\section{TEACHING POINTS:}

1. Restricted diffusion surrounding the surgical site may represent devitalized tumor tissue or infarct from retraction, vascular injury or devascularization. Large infarcts can result from sacrifice of tumor vascular supply that is shared by normal brain parenchyma.

2. Such areas may subsequently enhance and should not be mistaken for tumor progression 
Case 2 


\section{CASE 2: Prolonged lethargy following Meningioma Resection}

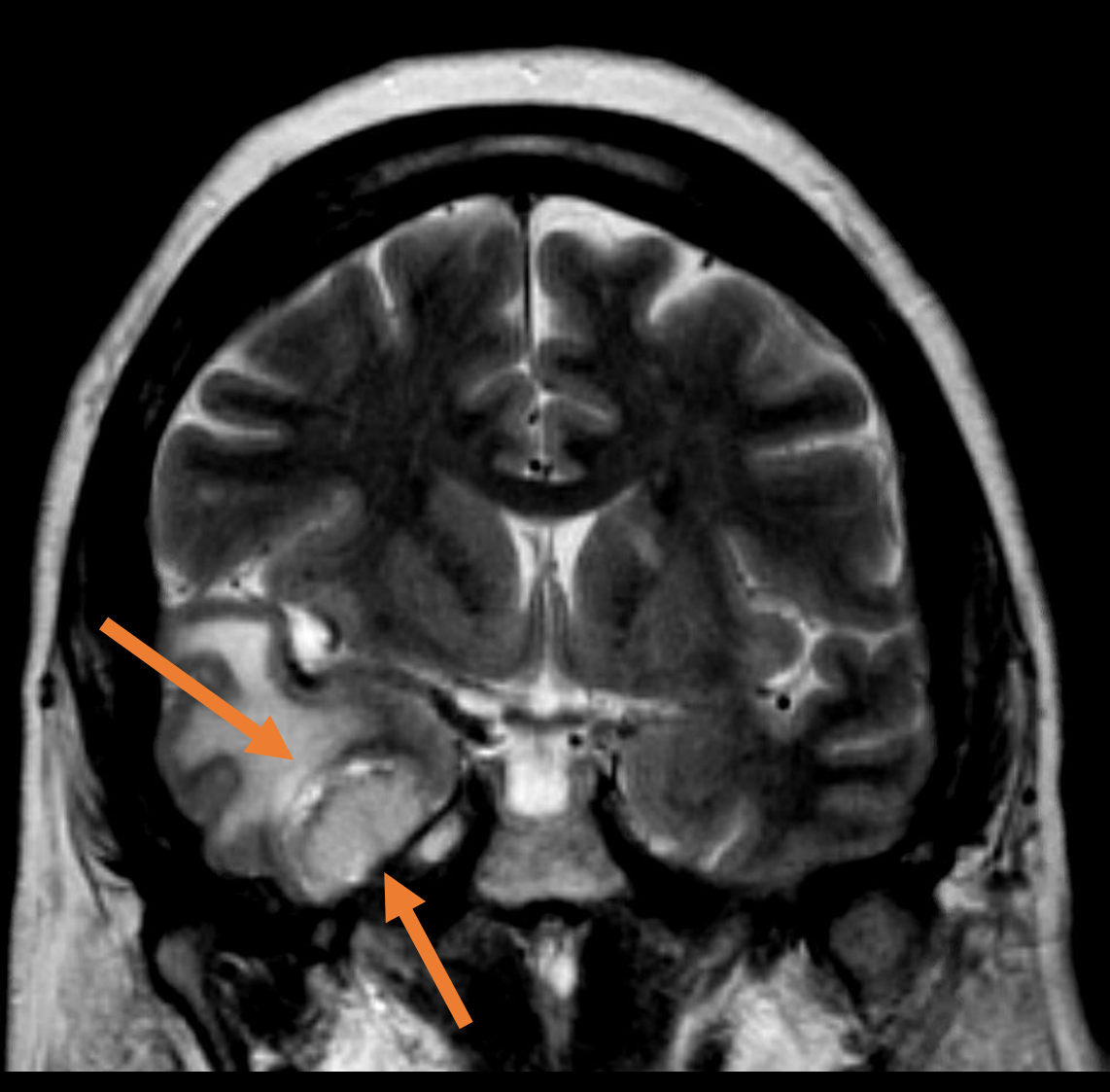

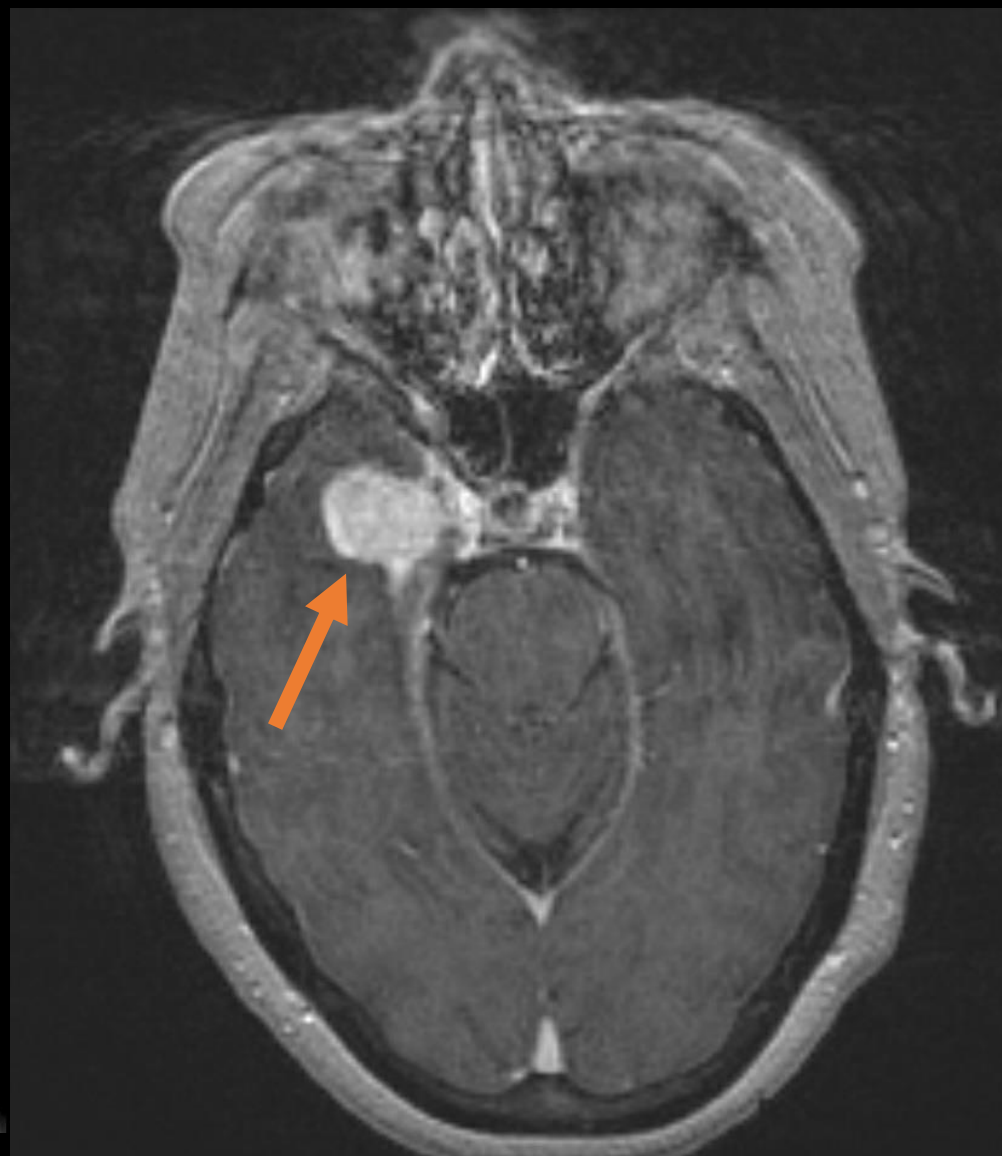

Axial T1 post
- History: 56 year old female with sudden onset transient right facial nerve palsy

- Pre-op imaging: Right middle cranial fossa meningioma with surrounding vasogenic edema.

- Surgery: Meningioma resection via right pterional craniotomy

- Of note, mannitol administration, hyperventilation and head elevation were performed for brain relaxation

\section{Post-op course:}

- Prolonged lethargy and delayed awakening

- EEG showed prolonged subclinical seizures, thought to originate from the right temporal lobe.
TEACHING POINT: Brain relaxation maneuvers decrease volume of intracranial contents (parenchyma, CSF, cerebral blood volume). They are used to minimize retraction injury and ischemia from compression. 


\section{Postoperative Unexpected Finding: Remote Cerebellar Hemorrhage}
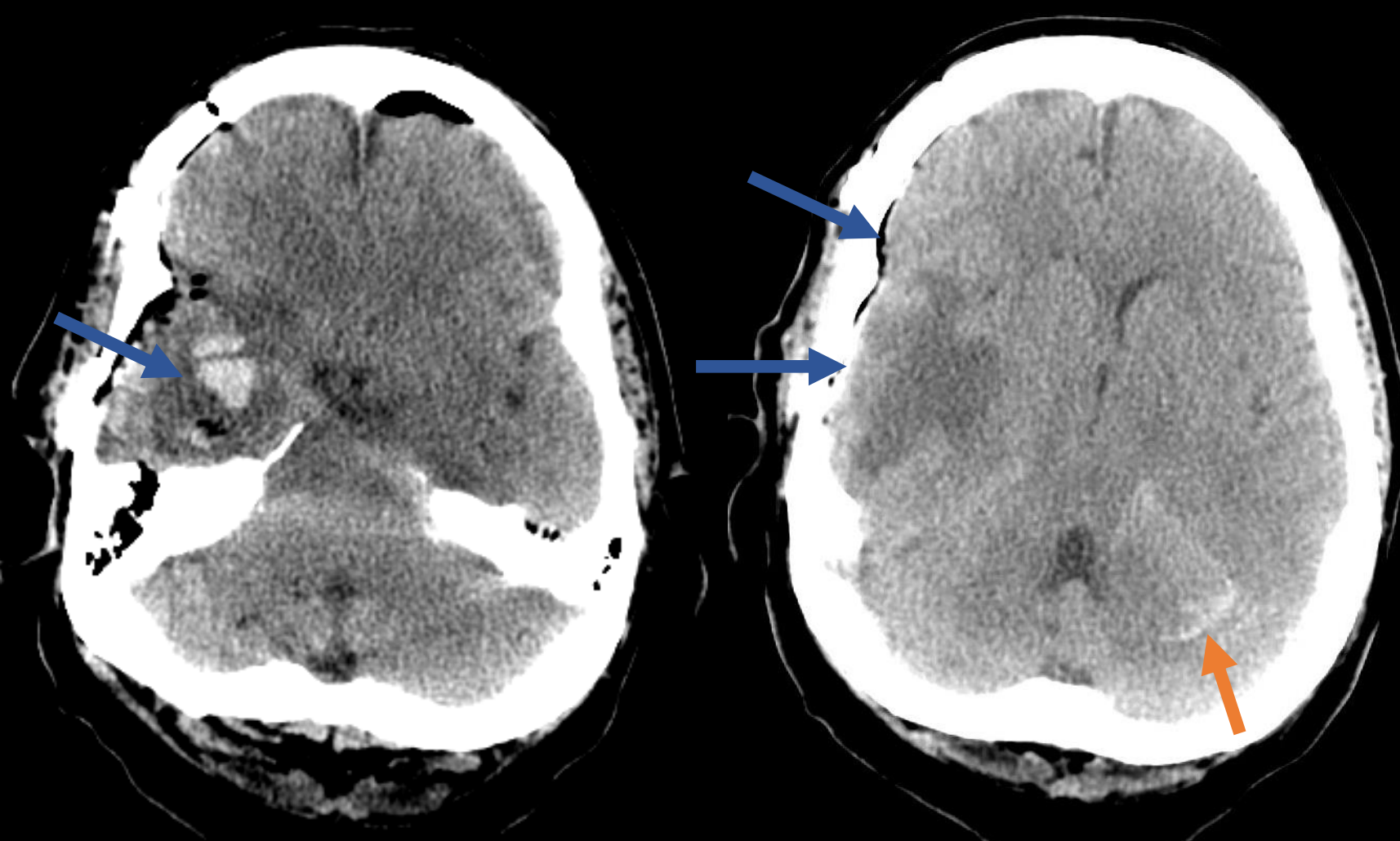

Immediate Post-op Non-contrast CT

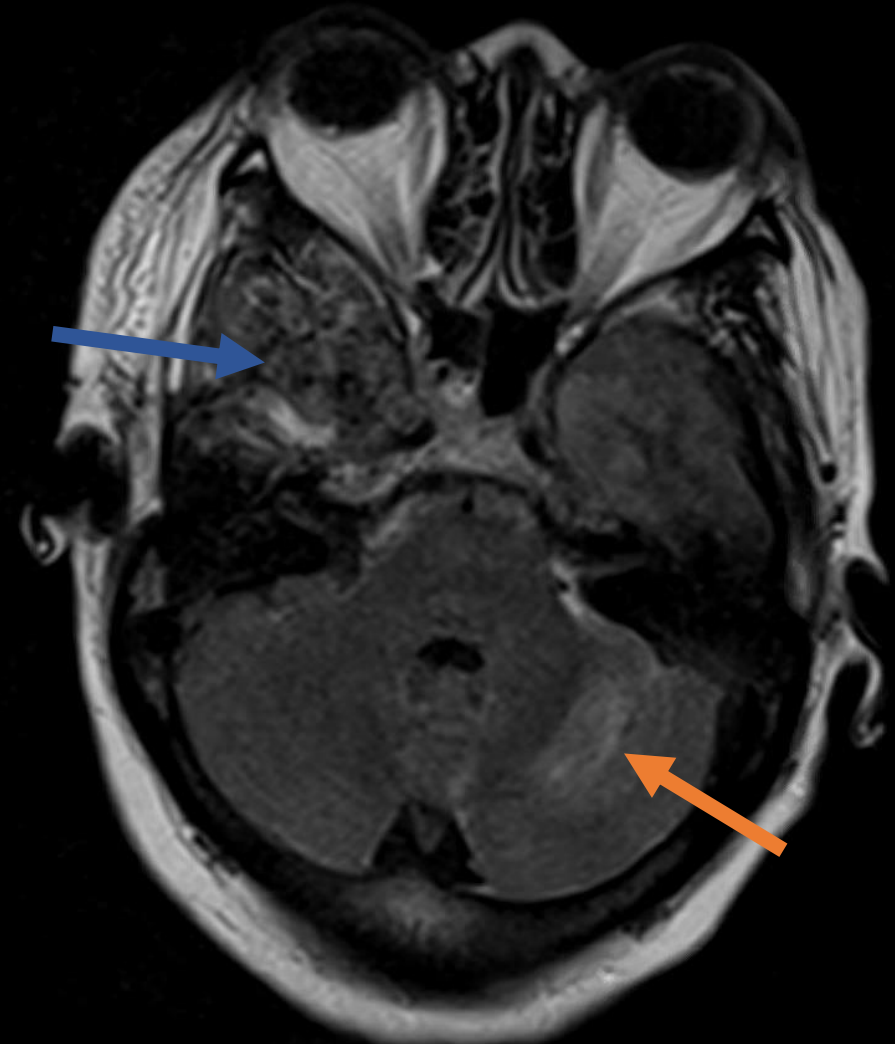

FLAIR

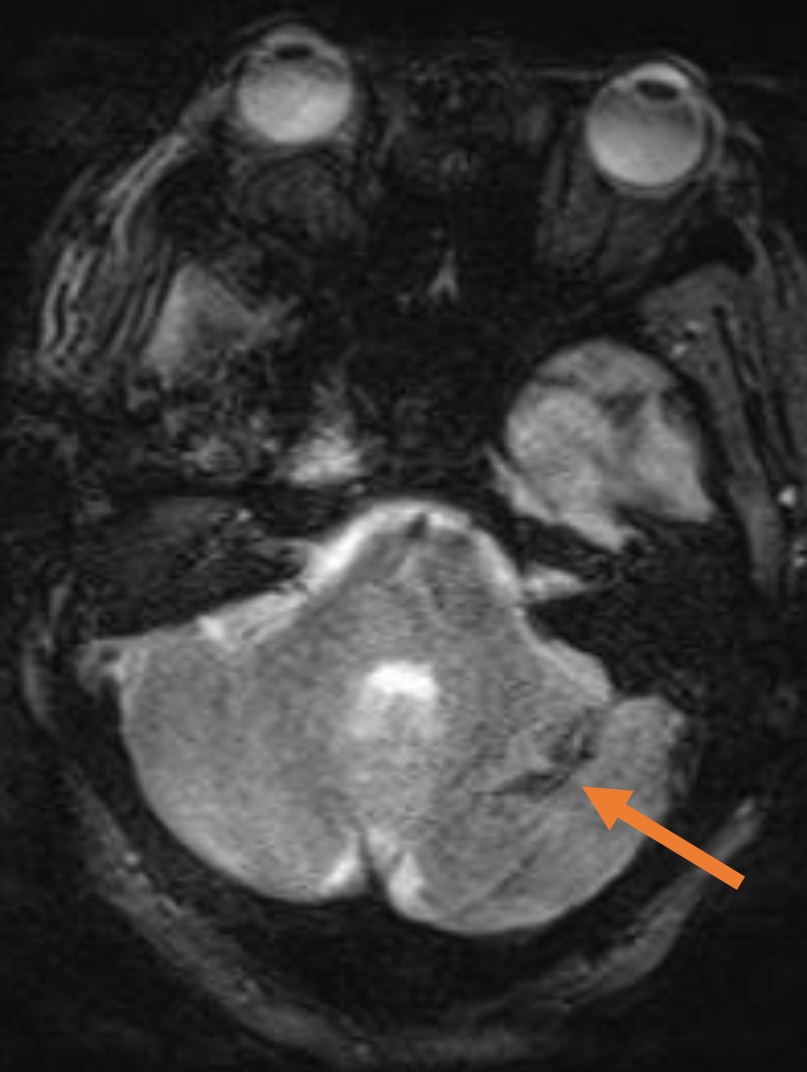

GRE

- Expected post-op changes at the surgical site: Scattered pneumocephalus, blood within the resection cavity with surrounding edema. Diffuse cerebral edema with leftward midline shift.

- Unexpected finding: Trace subarachnoid blood along the superior left cerebellum.

- Patient improved with expectant management and was discharged with minimal deficits

TEACHING POINT: Remote cerebellar hemorrhage is a predominantly self-limiting condition that occurs rarely following supratentorial craniotomies. It is thought to result from CSF hypovolemia and cerebellar shifting/sagging causing bridging vein tearing or occlusion. 
Case 3 


\section{CASE 3: New Alexia (Inability to read) Following Microvascular Decompression}

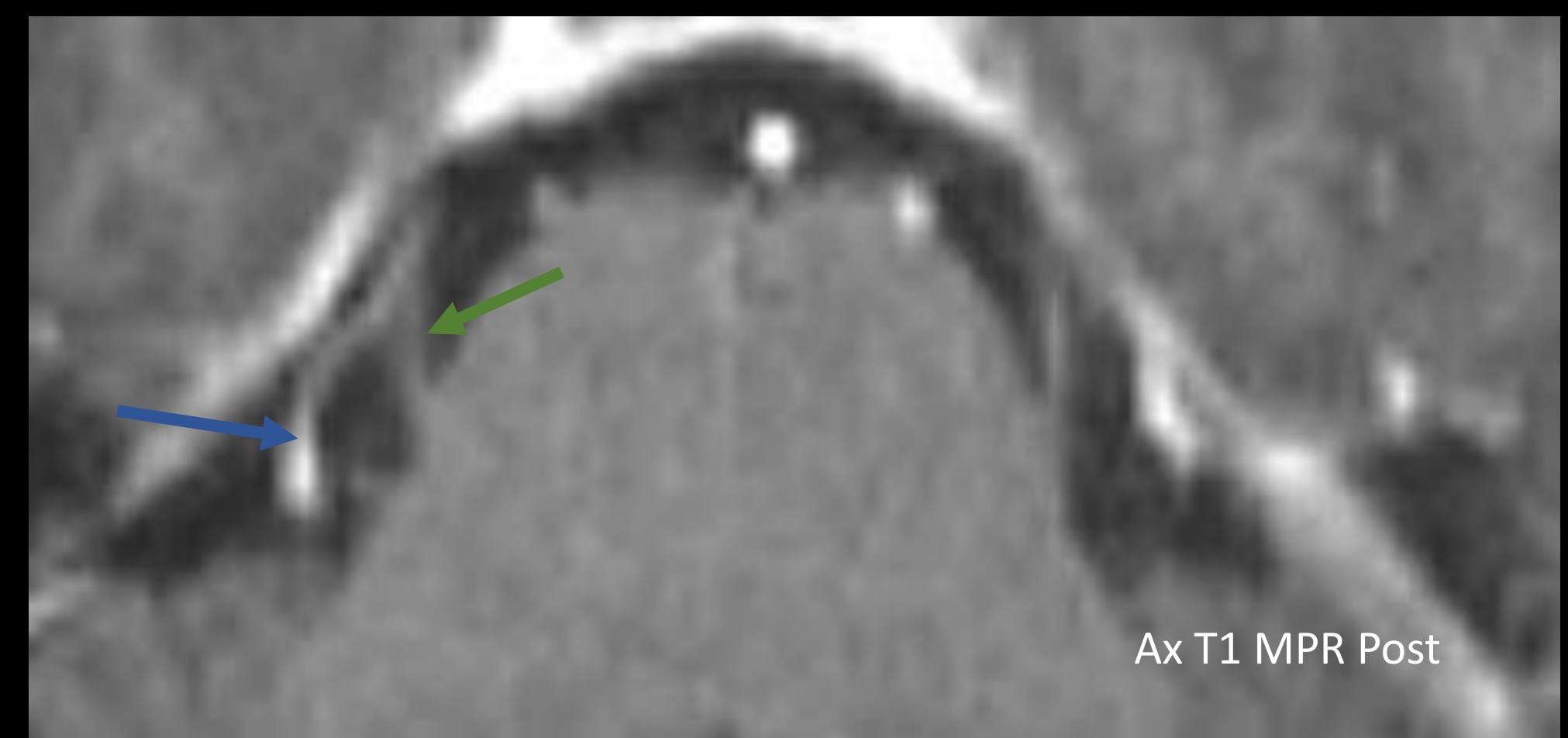

- History: 65-year-old male with 2-year history of right facial pain diagnosed with trigeminal neuralgia; failed medical management.

- Pre-op imaging: An artery (distal right superior cerebellar artery) coursing over the cisternal segment of the trigeminal nerve ipsilateral to the patient's symptoms, suggestive of vascular compression.

- Surgery: Microvascular decompression of trigeminal nerve, Suboccipital approach.

- Post-op Course:

- Difficult to wake up post operatively requiring intensive airway monitoring

- New aphasia and difficulty reading and calculating on POD1, which prompted emergent CT 


\section{Postoperative Unexpected Finding: Remote Intraparenchymal Hemorrhage}
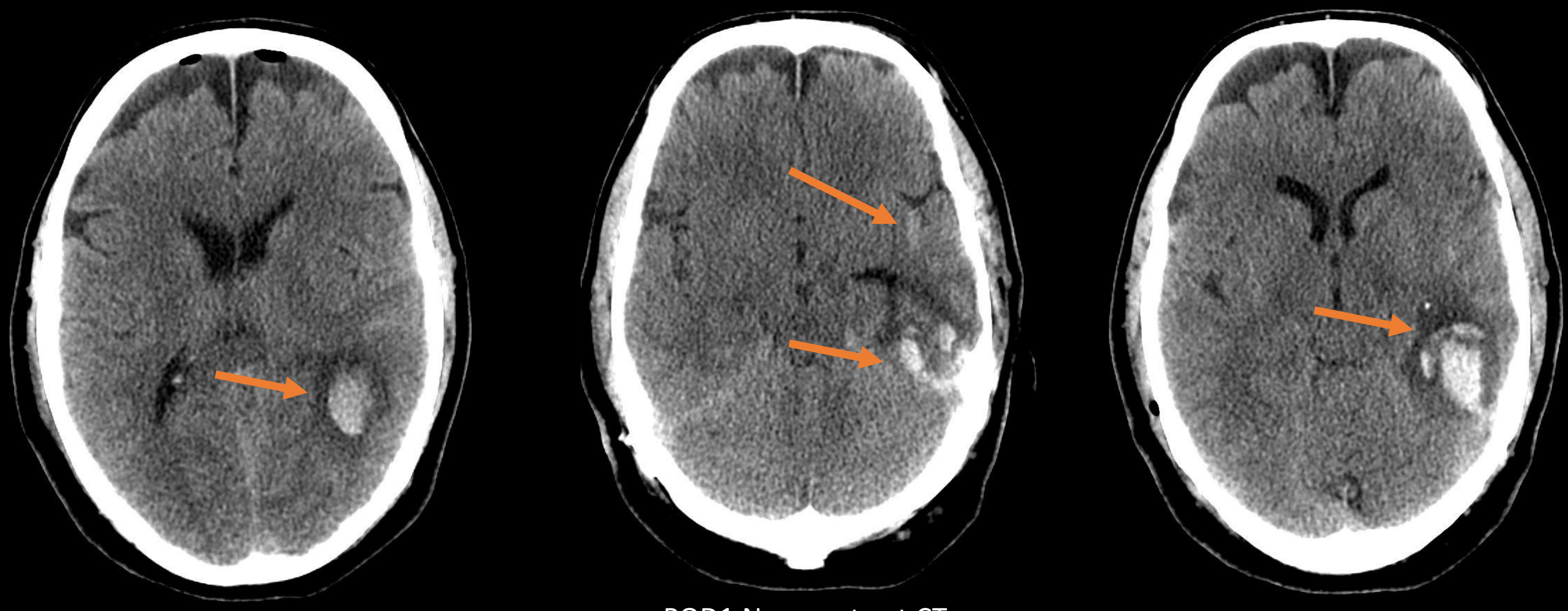

POD1 Non-contrast CT

- Unexpected finding: New left posterior temporal intraparenchymal hematoma with subarachnoid extension, remote in location to the site of surgery. This finding is likely due to venous stretch injury from CSF shift

- Patient was discharged on POD6 with mild residual aphasia and alexia

\section{TEACHING POINTS:}

1. Remote intraparenchymal hemorrhages from CSF shift can occur both supra and infra-tentorially.

2. Intraparenchymal hemorrhage has been reported up to $10.8 \%$ of post operative patients, with a majority being small, under $3 \mathrm{~cm}$. 
Case 4 
- History: 46-year-old female with 21-year history of medically-refractory seizures

- Pre-op imaging: Left hippocampal atrophy with increased T2/FLAIR signal and loss of internal architecture, consistent with mesial temporal sclerosis
- Surgery: MRI-guided stereotactic left temporal laser ablation

- Intra-op MRI findings: Following stylet advancement under stereotactic guidance, there is new acute blood products within the basal cisterns

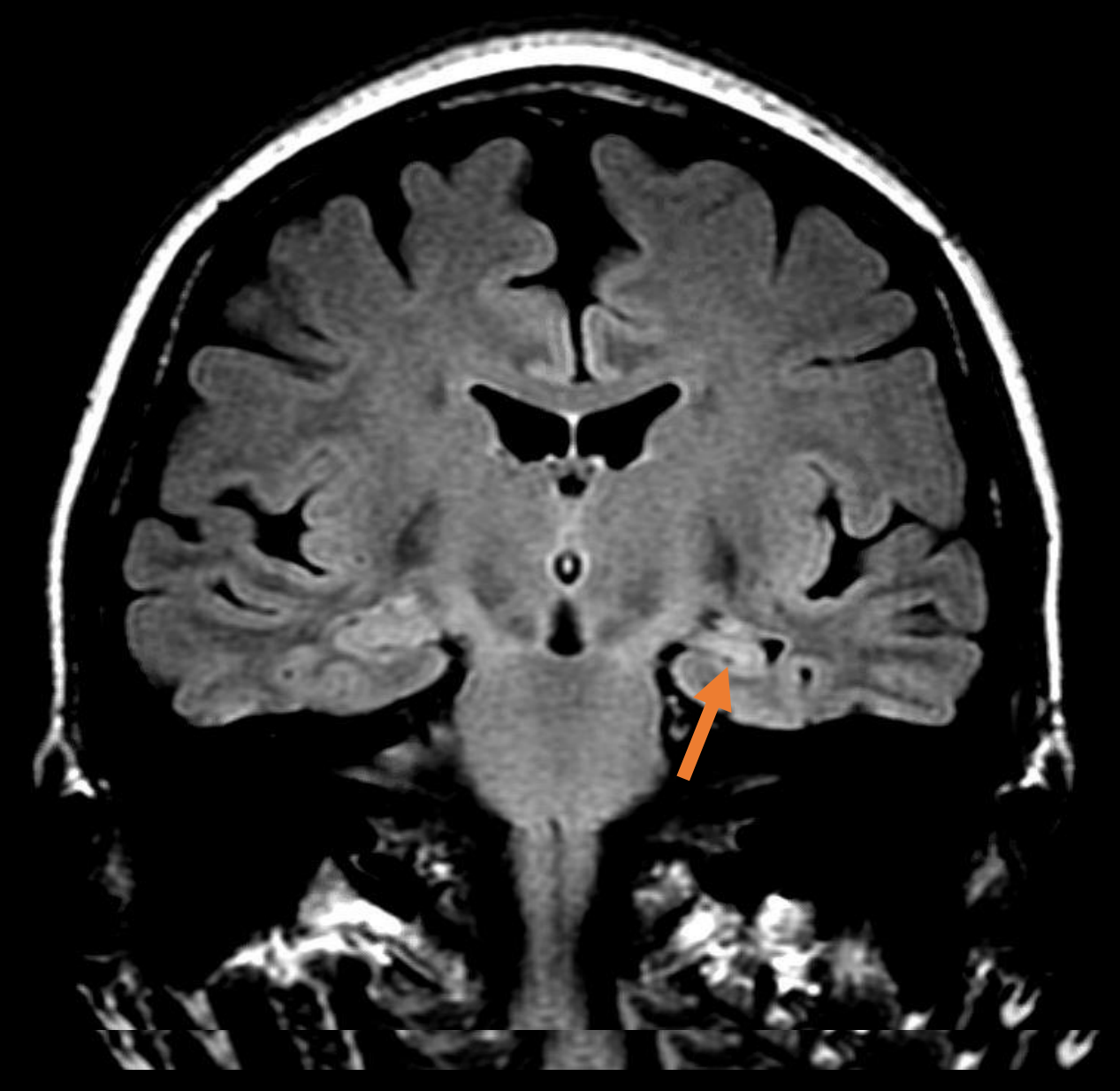

Coronal FLAIR

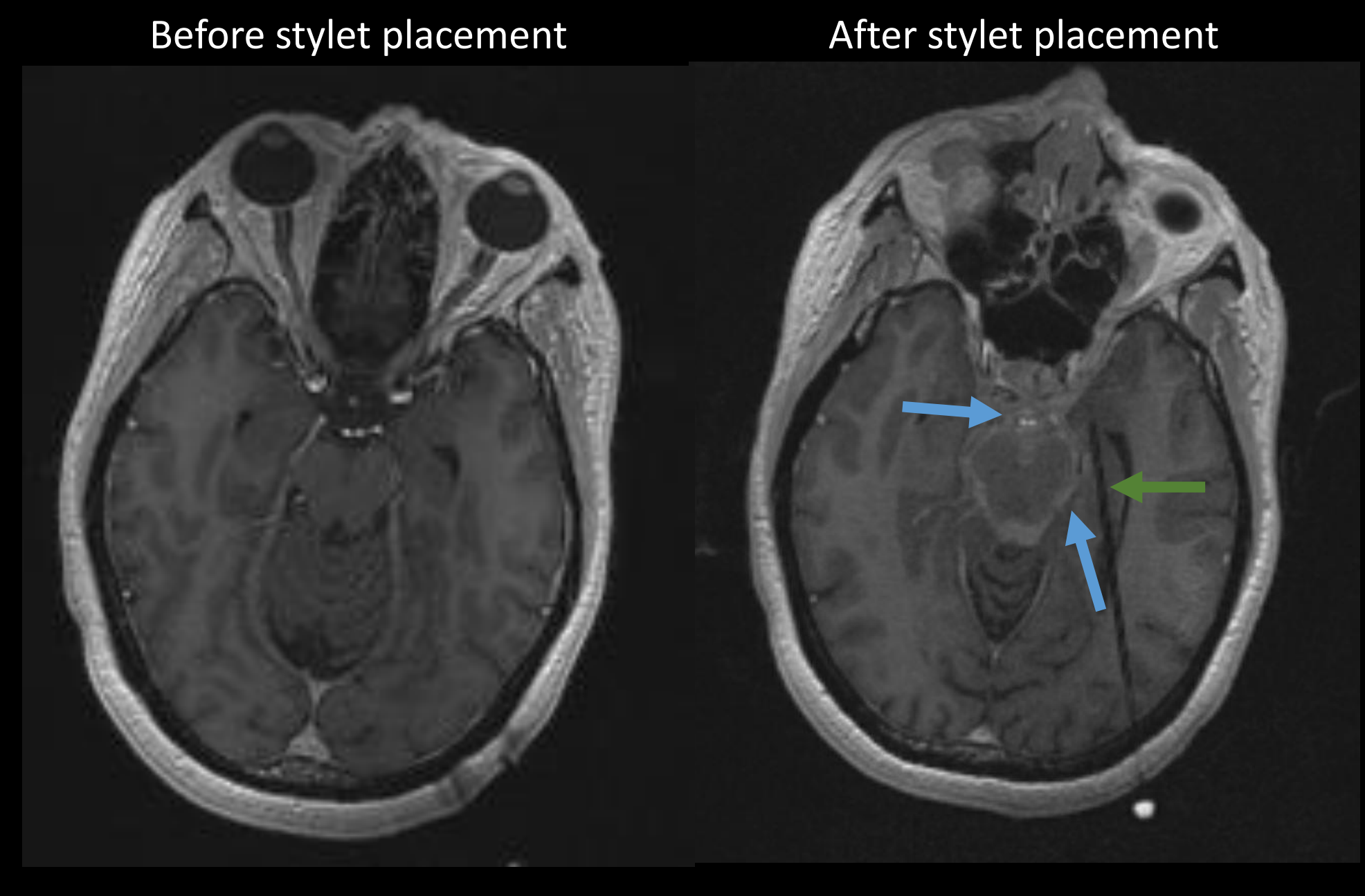

T1 Post-contrast 
- Procedure was aborted. Emergent angiography (with stylet still in place) showed no source for bleeding. Emergent CT showed stable basal cisternal subarachnoid hemorrhage. Decision was made to resume left temporal laser ablation.

- Subsequent Intra-Op MRI findings: Ablation stylet was exchanged for the ablation catheter with completion of ablation. There was progressive worsening intracranial hemorrhage with subarachnoid hemorrhage in the basal cisterns, left sylvian fissure, and intraventricular extension into the left lateral ventricle

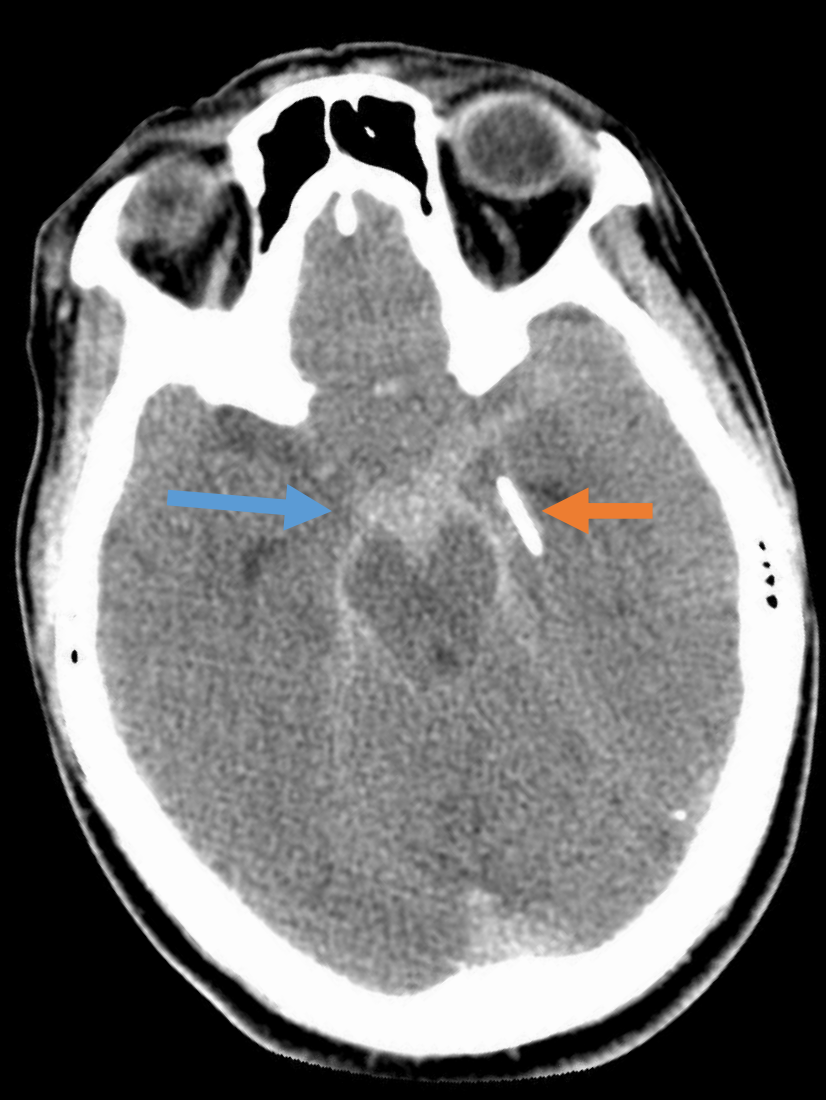

Emergent CT after angiography

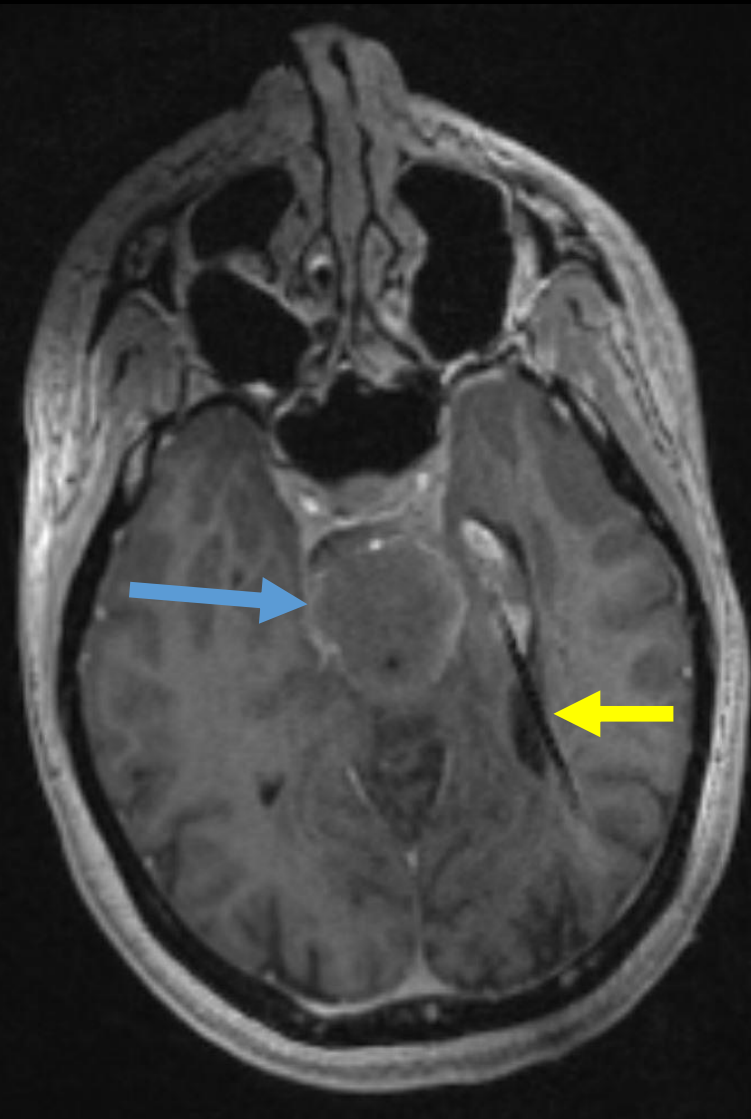

T1 Post-contrast During ablation
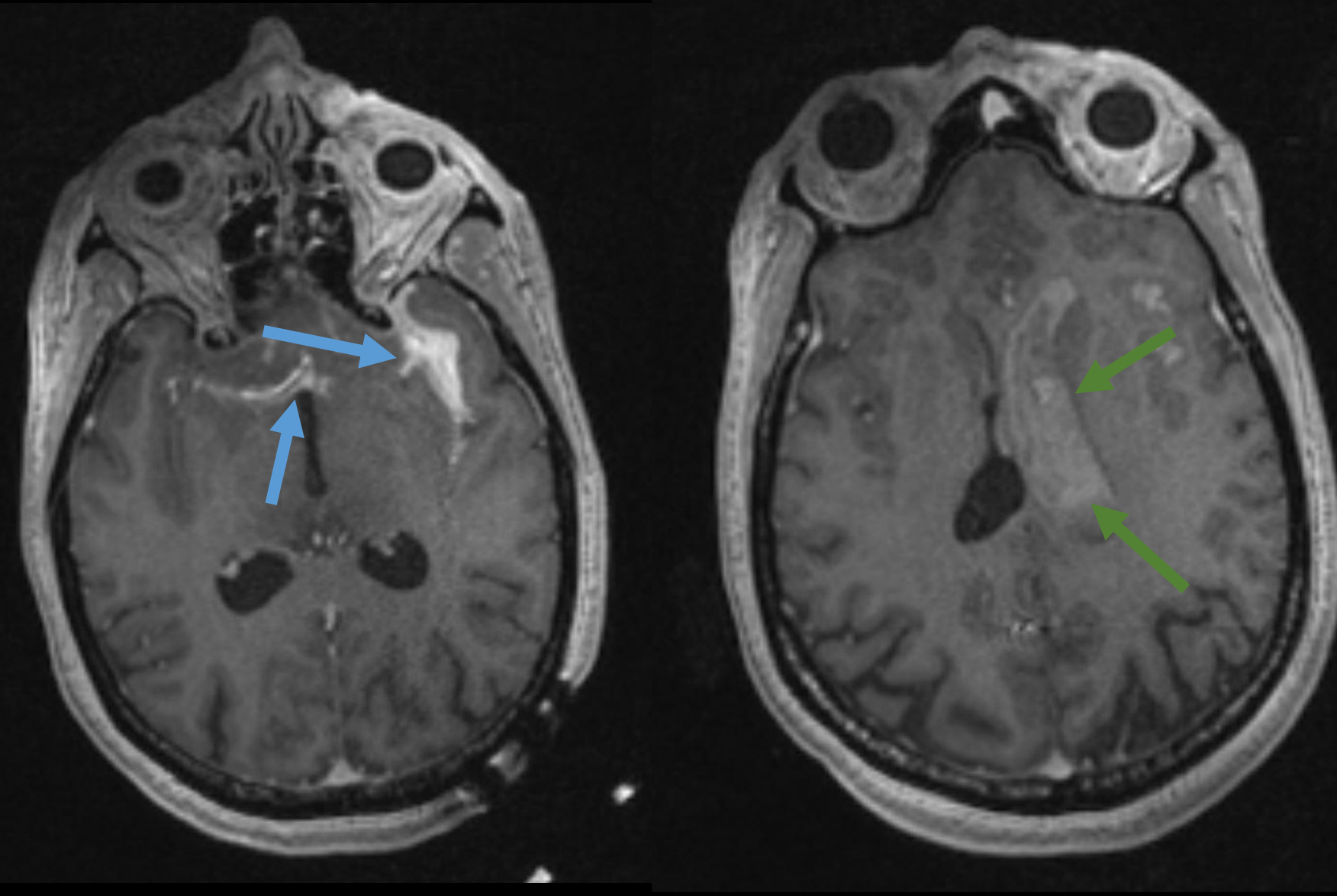

T1 Post-contrast, after ablation was completed 


\section{Postoperative Unexpected Finding: Pseudoaneurysm along ablation catheter tract}

- CTA performed on POD5 for vasospasm follow-up showed a hyperdense focus adjacent to the left P2 segment, confirmed to be a $3 \mathrm{~mm}$ PCA perforator fusiform pseudoaneurysm on angiogram. Repeat angiogram 4 weeks later following conservative management demonstrated resolution.
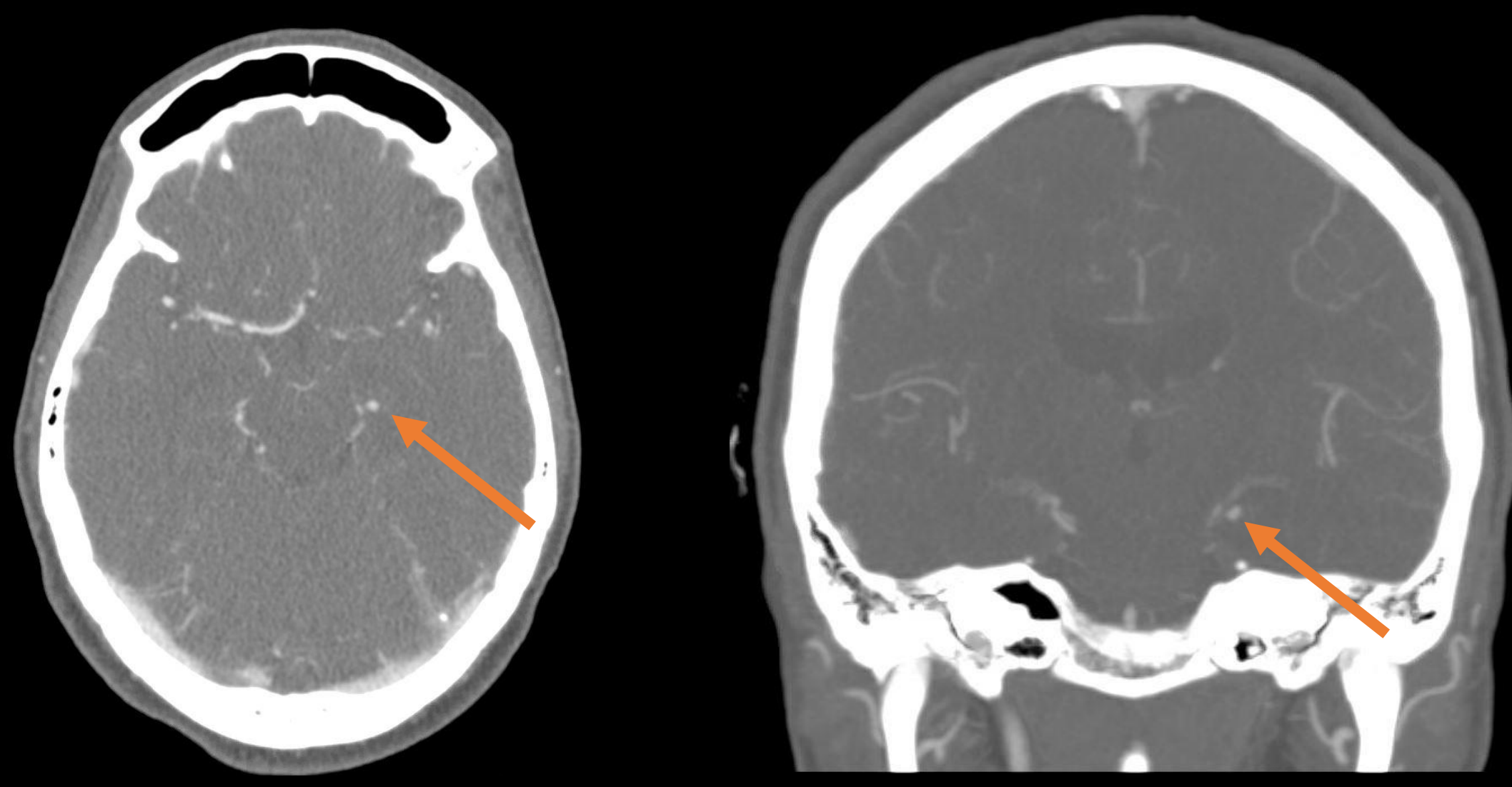

POD 5 CTA

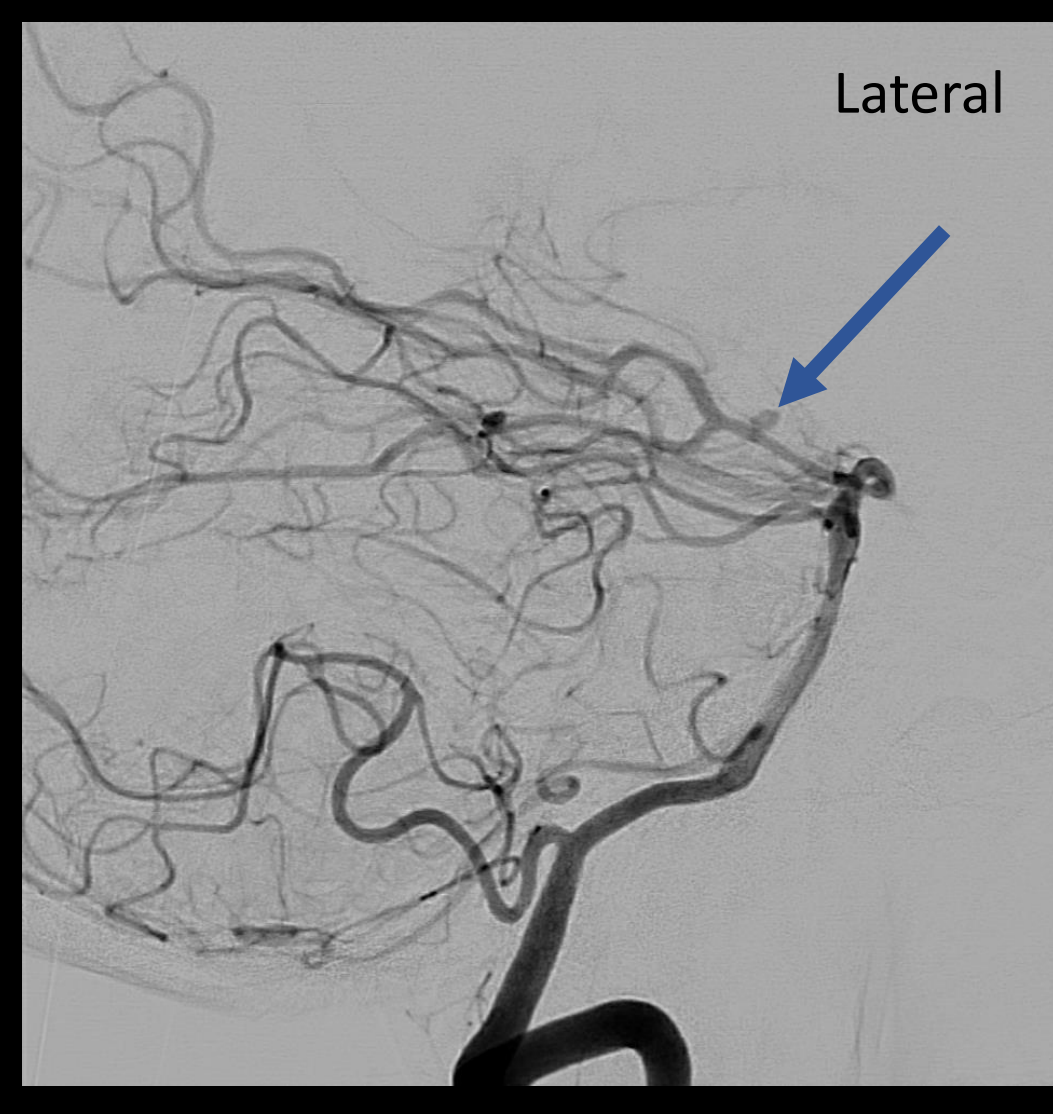

POD 5 DSA, left vertebral injection

TEACHING POINT:

1. Despite stereotactic guidance, neurosurgical devices such as stylets/catheters can injure nearby vessels, causing hemorrhage and/or strokes .

2. It is crucial to scrutinize the entirety of the device tract/trajectory for potential injury. 
Case 5 
- $\underline{\text { History: }}$ 62-year-old male transferred to our institution for further management after excessive intra-op bleeding necessitating emergent packing 1 day prior
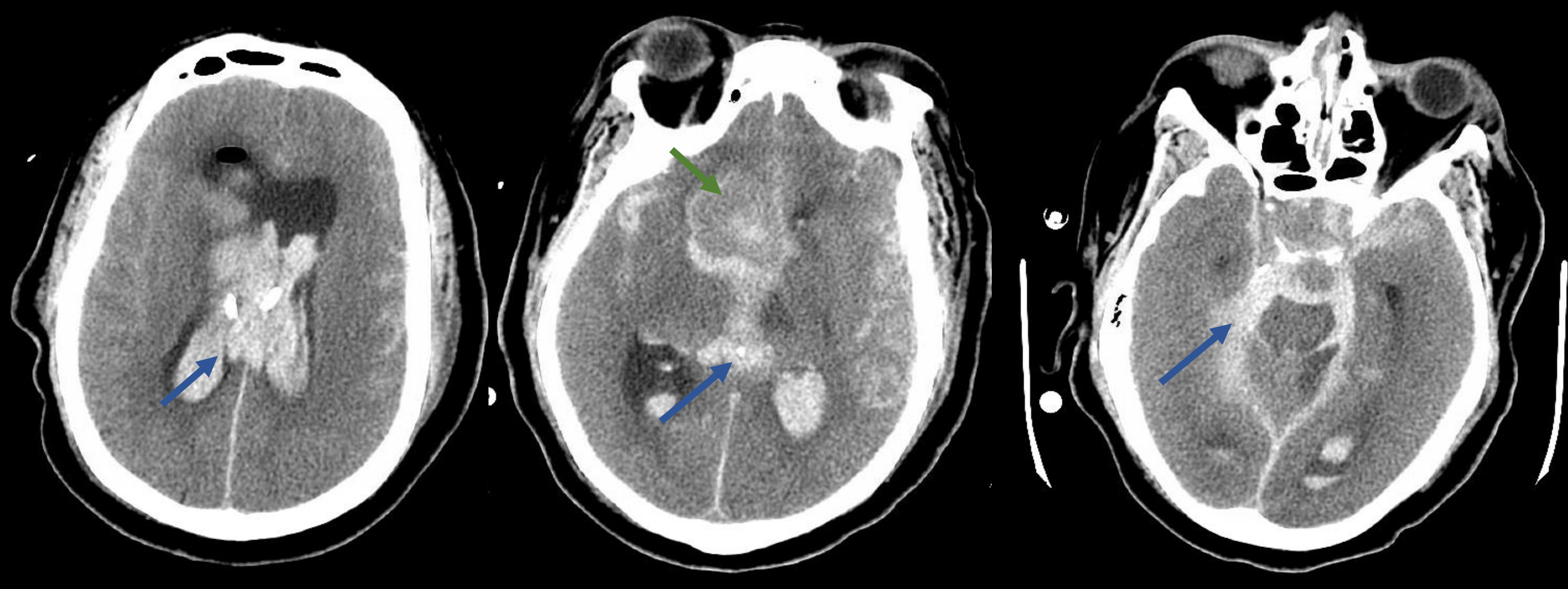

POD1 Non-contrast CT

- Surgery: Transsphenoidal resection of pituitary macroadenoma.

- Post-op imaging: Extensive subarachnoid hemorrhage with intraventricular extension and acute hydrocephalus. Residual pituitary macroadenoma is noted. 
Device Related Complications 
Case 6 


\section{Case 6: Complex Clipping of Unruptured Left ICA Aneurysms}

- History: 58-year-old female with multiple unruptured aneurysms found on tinnitus workup

- Pre-op imaging: Left ICA angiogram revealed two left ophthalmic ICA aneurysms
- Surgery: Complex clipping of ICA aneurysms via left pterional craniotomy

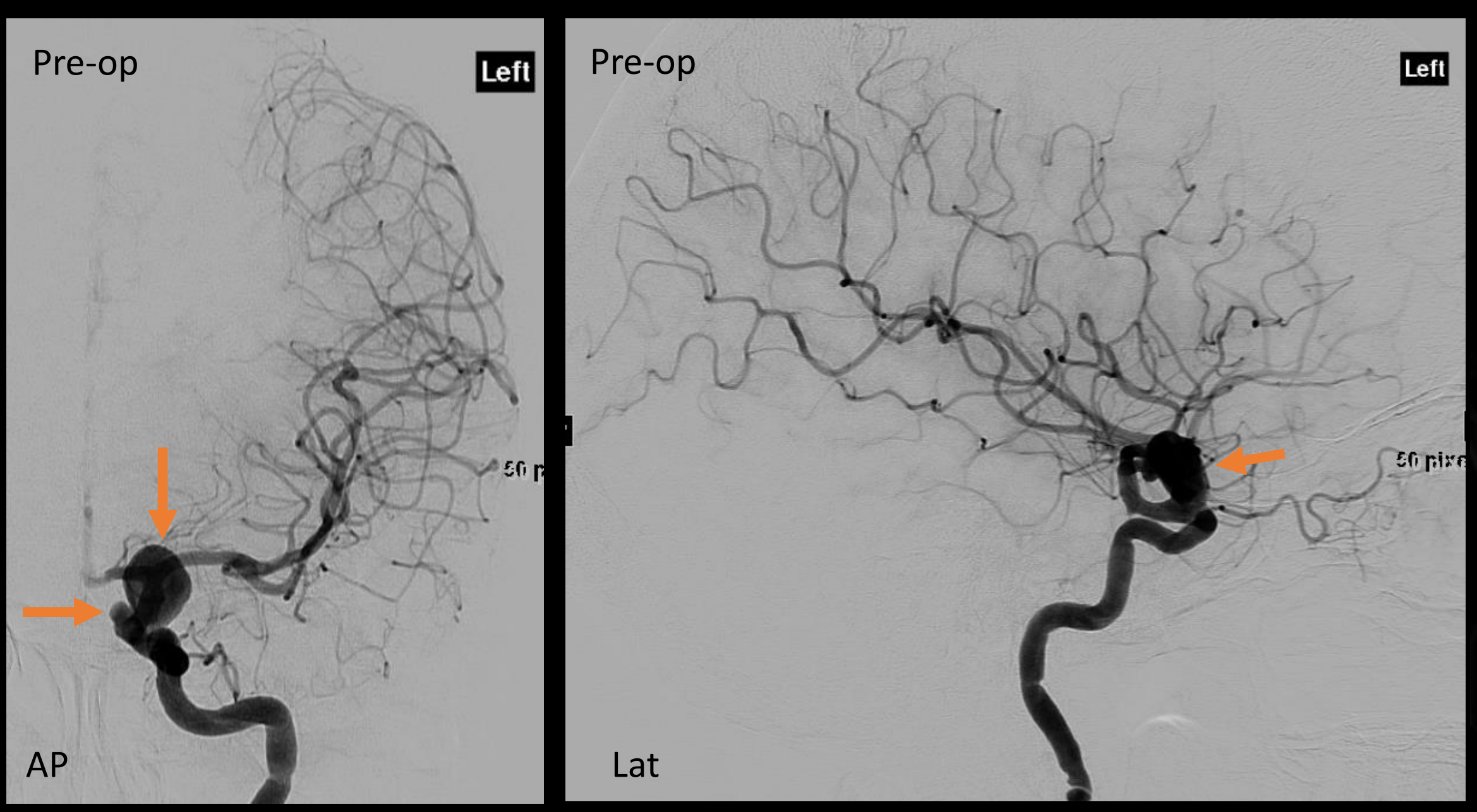

POD1 Non-contrast CT

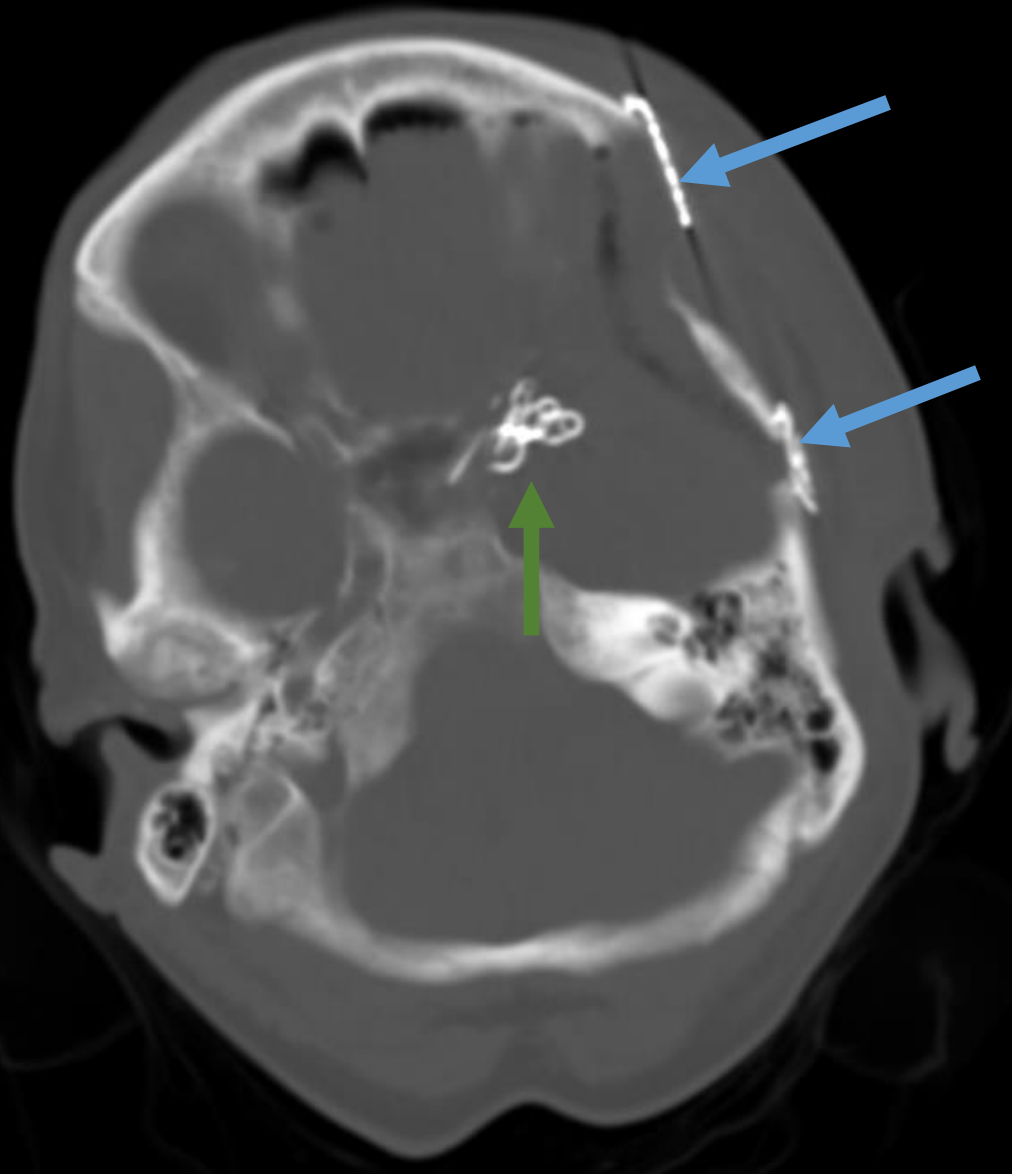

- Post-op Imaging findings: Several aneurysm clips with expected post craniotomy appearance with titanium plates in place. There is no residual aneurysm filling (not shown). 
Late Post-Operative Unexpected Finding: Delayed seizures with Left Temporal Enhancement

Clinical Course: Patient developed new partial seizures 9 months following surgery, prompting repeat MRI

Cor T1 Post-Contrast

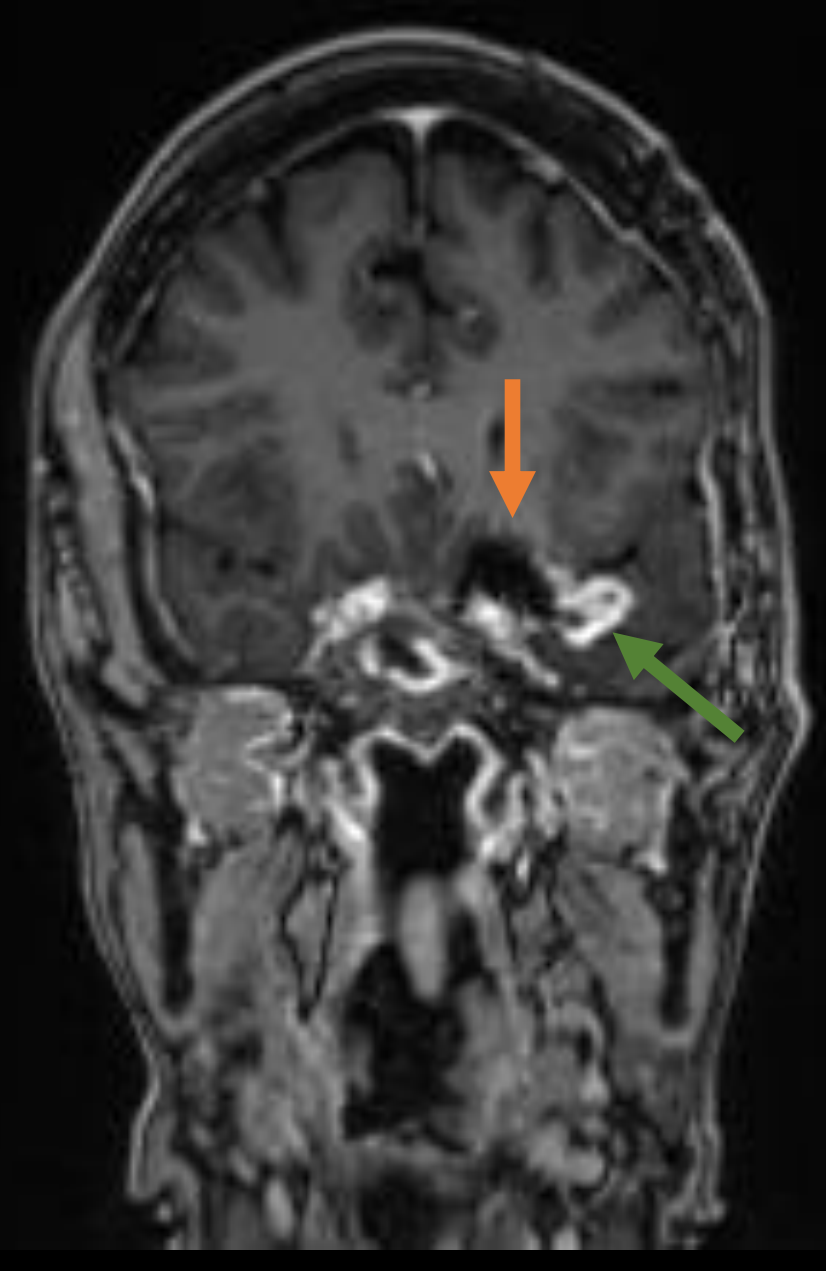

Ax T1 Post-Contrast

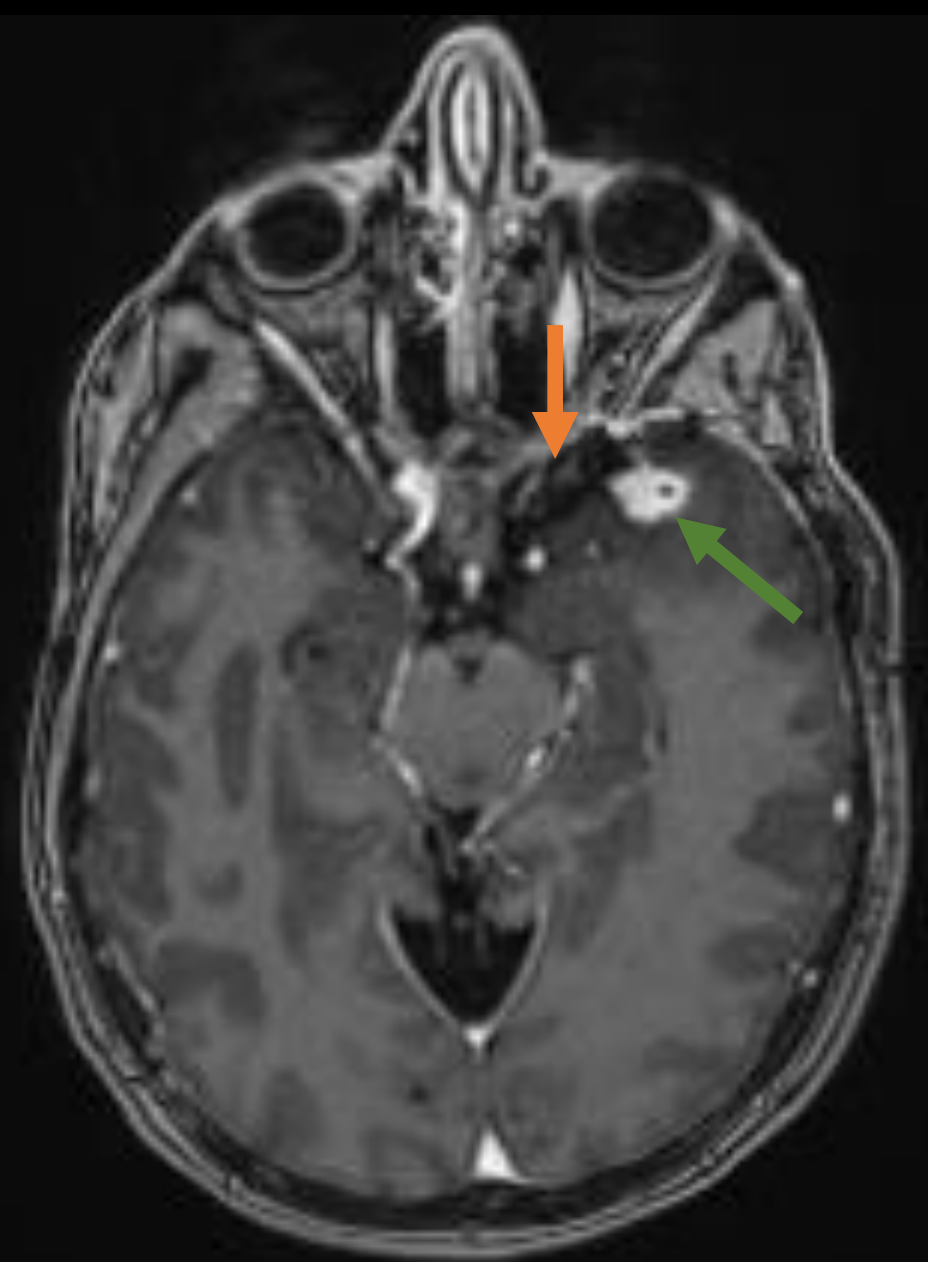

Ax FLAIR

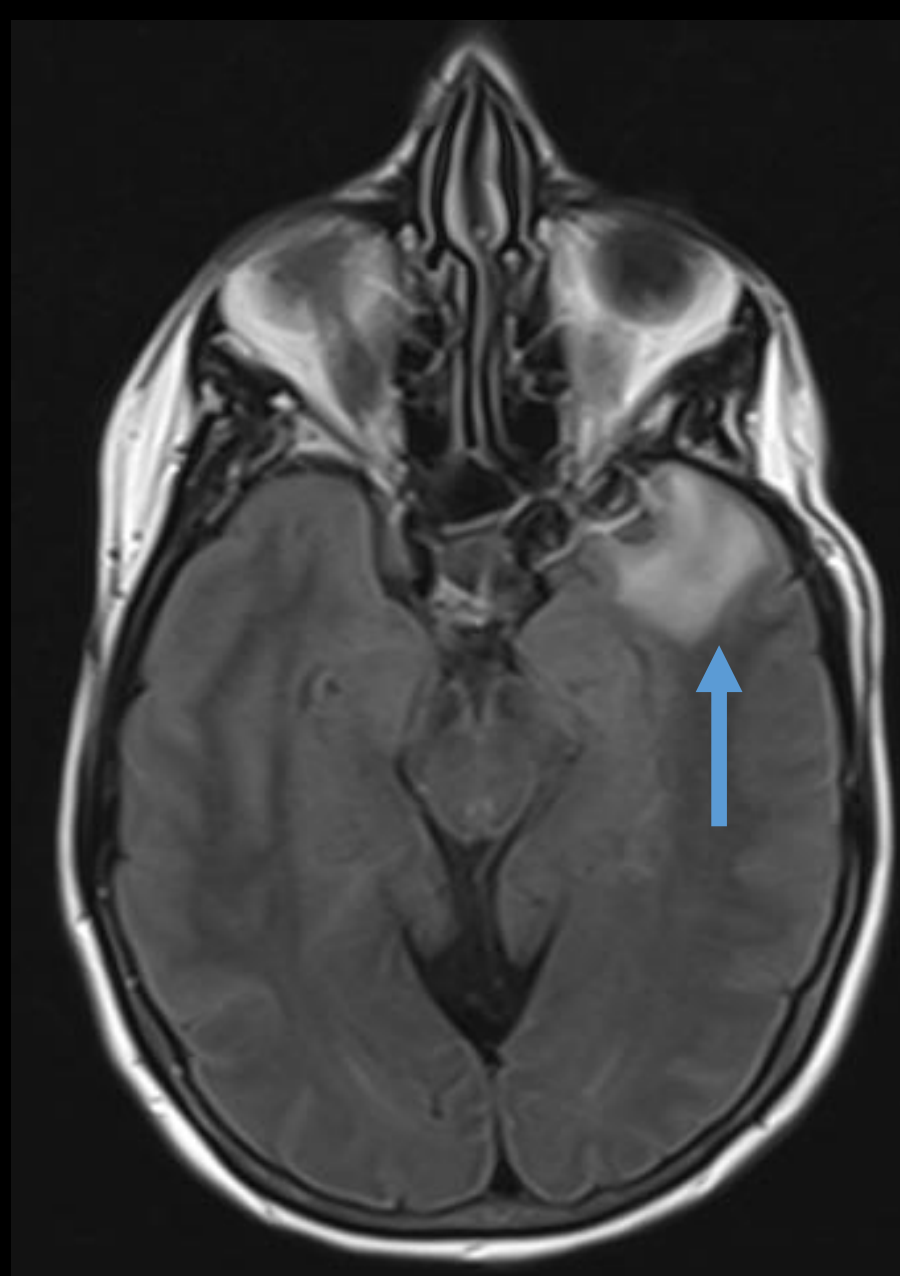

Imaging findings: New left temporal pole enhancing lesion along the susceptibility artifact from aneurysm clips, with surrounding edema. Findings initially worsened then improved slightly over the subsequent 2 years. 


\section{Late Post-Operative Unexpected Finding: Granulomatous reaction to Muslin (Muslinoma)}

DSA was performed to evaluate for aneurysm leak, recurrence, or pseudoaneurysms, which revealed clipped aneurysms with unchanged remnant neck.

Diagnosis was made of inflammatory reaction to muslin, which was used for aneurysm wrapping during initial procedure. Patient remained clinically stable without need for further interventions.
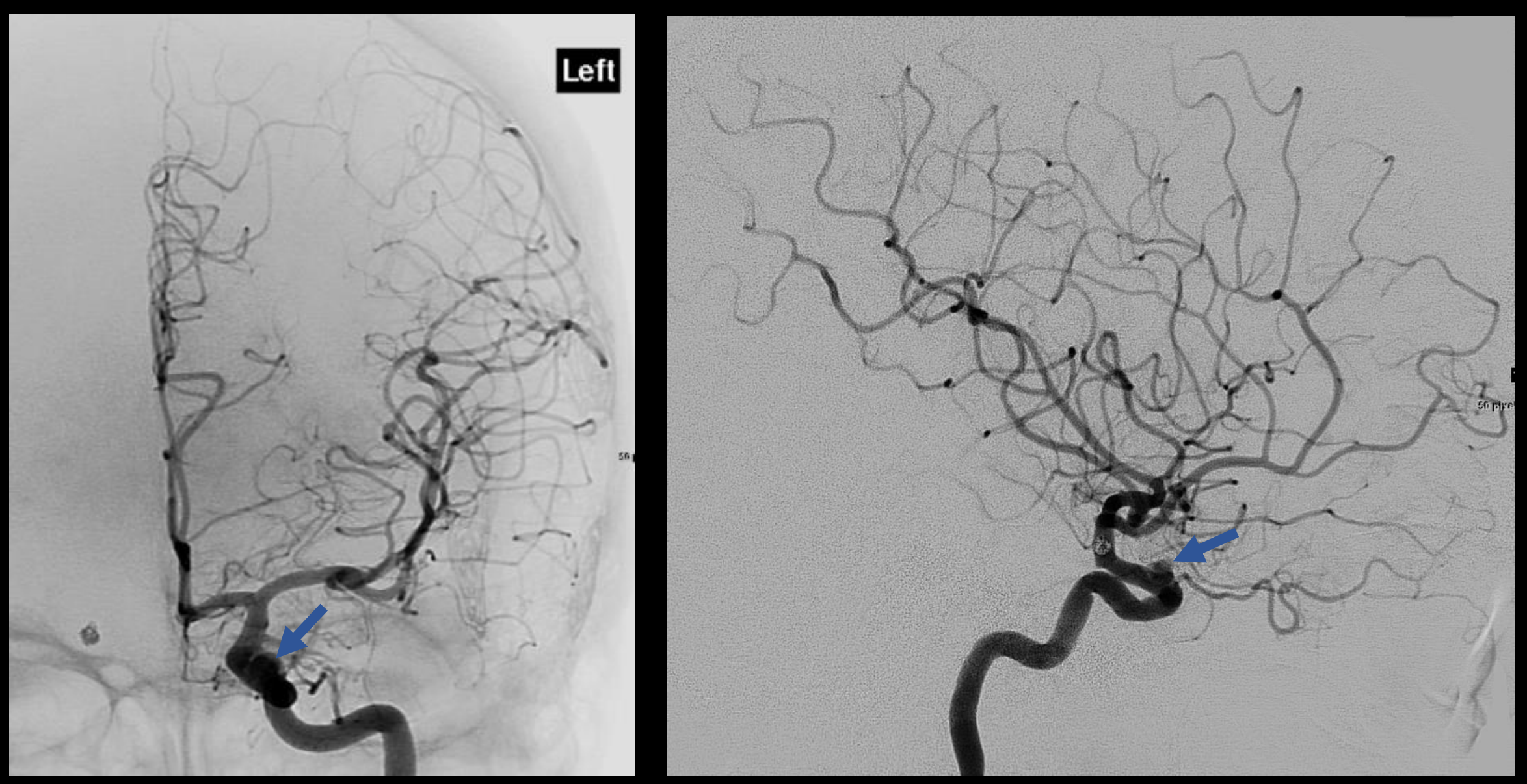

\section{TEACHING POINTS:}

1. Aneurysm wrapping with muslin or cotton gauze reinforces the aneurysm, and can be used in conjunction with clips to treat complex aneurysms

2. Resultant delayed (months to years) foreign body inflammatory reaction can result in a perianeurysmal rim-enhancing mass (muslinoma or gauzoma) with surrounding edema, with no diffusion restriction.

3. Other complications related to muslin usage include optic neuropathy, adhesive arachnoiditis, sterile intracranial abscesses, and parent artery narrowing 
Case 7 


\section{CASE 7: Right ICA Pipeline Stent Placement for Ophthalmic Artery Aneurysm}

- History: 63-year-old female with previously coiled ruptured left PICA aneurysm, now presents for treatment of unruptured right ophthalmic artery aneurysm

- Pre-op imaging: DSA and MRI show a wide neck right ophthalmic artery aneurysm

- Procedure: Right ICA Pipeline Stent placement
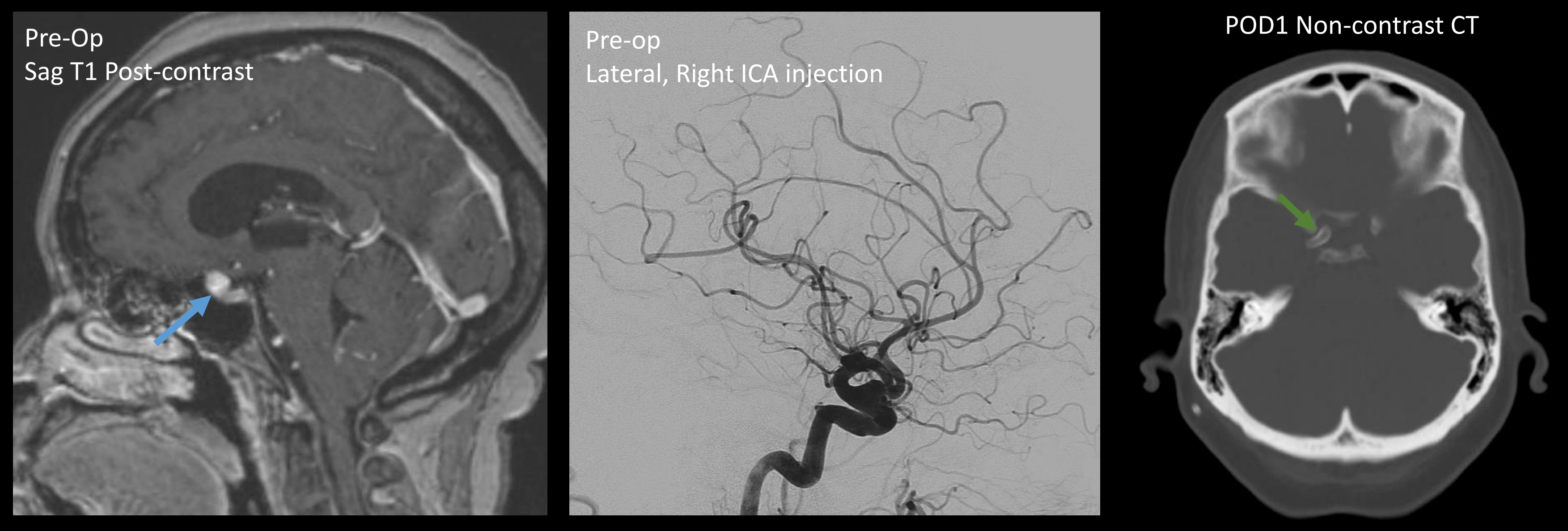

- Post-op imaging: NCECT shows expected position of the right ICA pipeline stent 


\section{CASE 7: New Seizures 1 month After Right ICA Pipeline Stent Placement}

- Clinical course: Patient was readmitted for development of new seizures 1 month post operatively

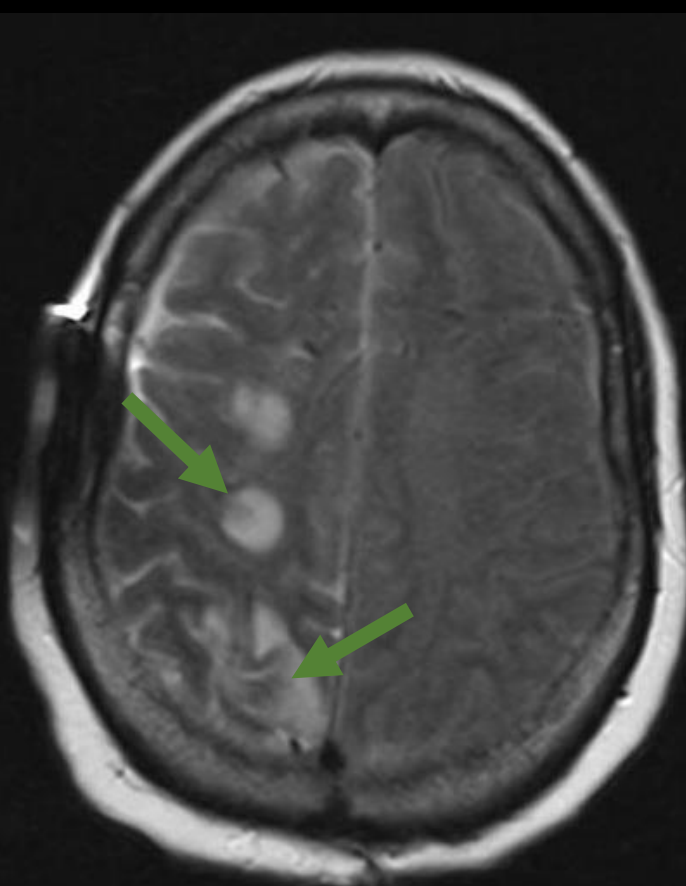

FLAIR

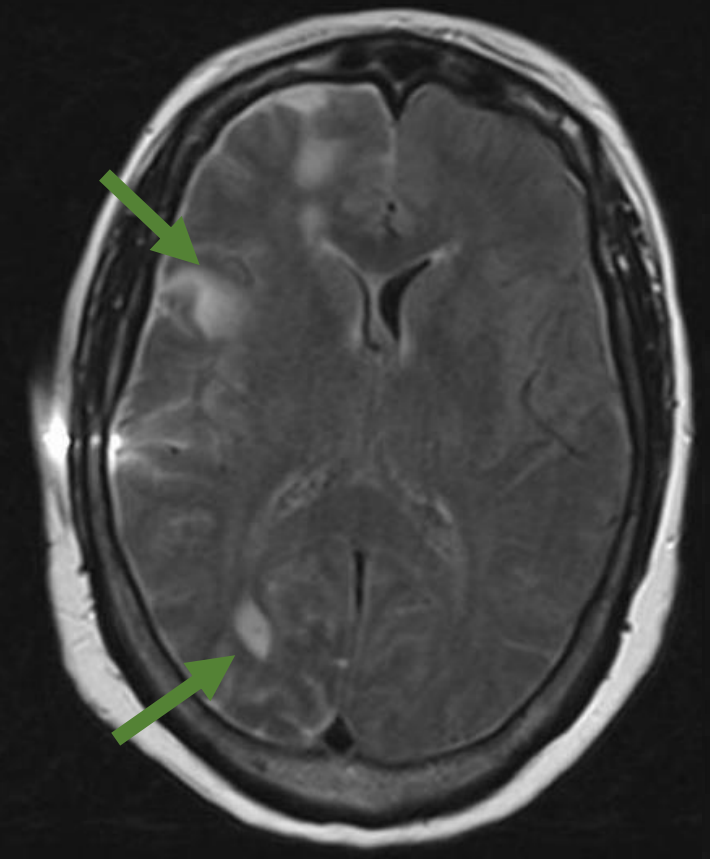

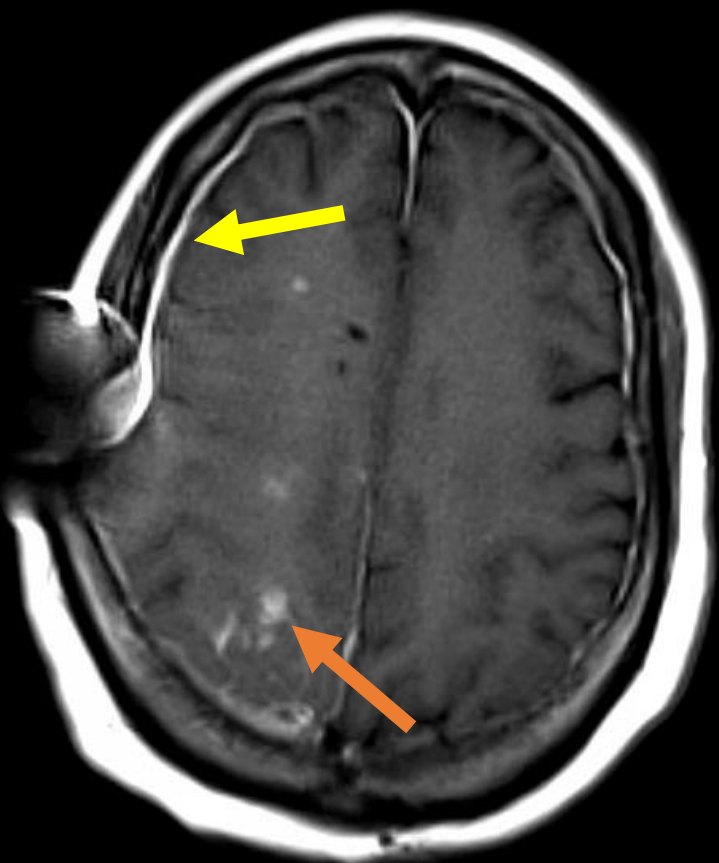

T1 Post-contrast

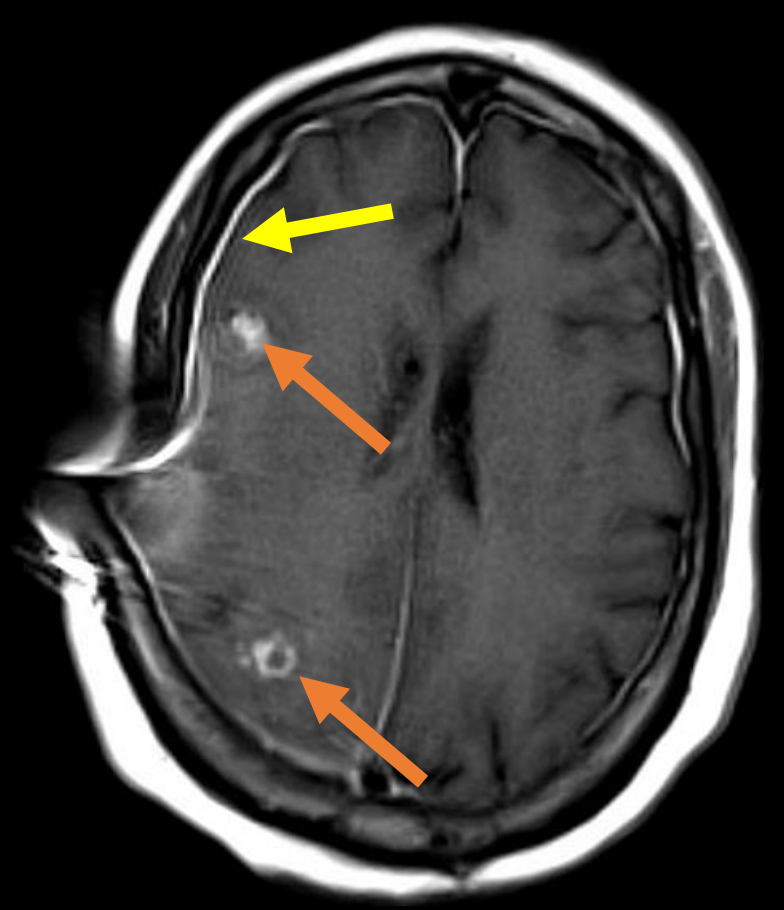

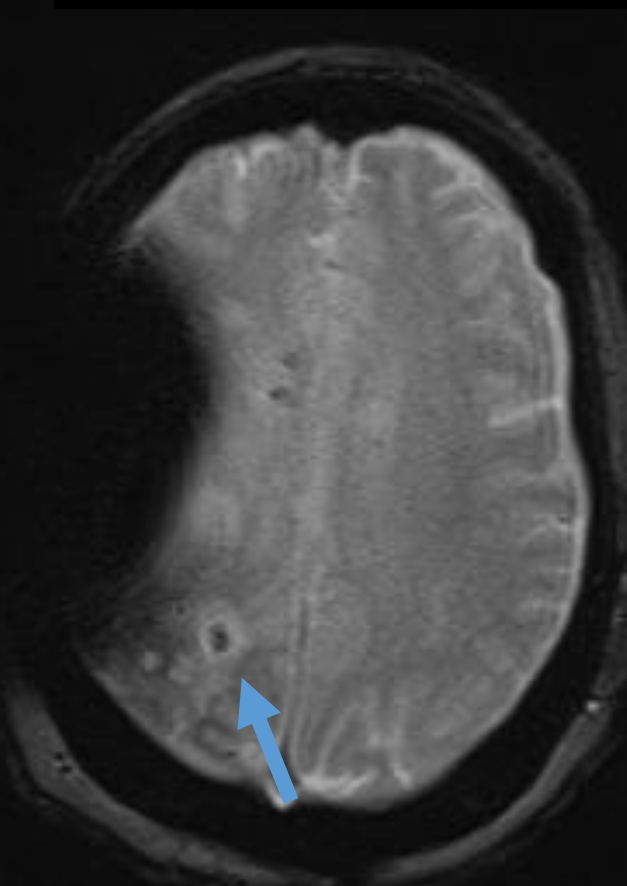

GRE

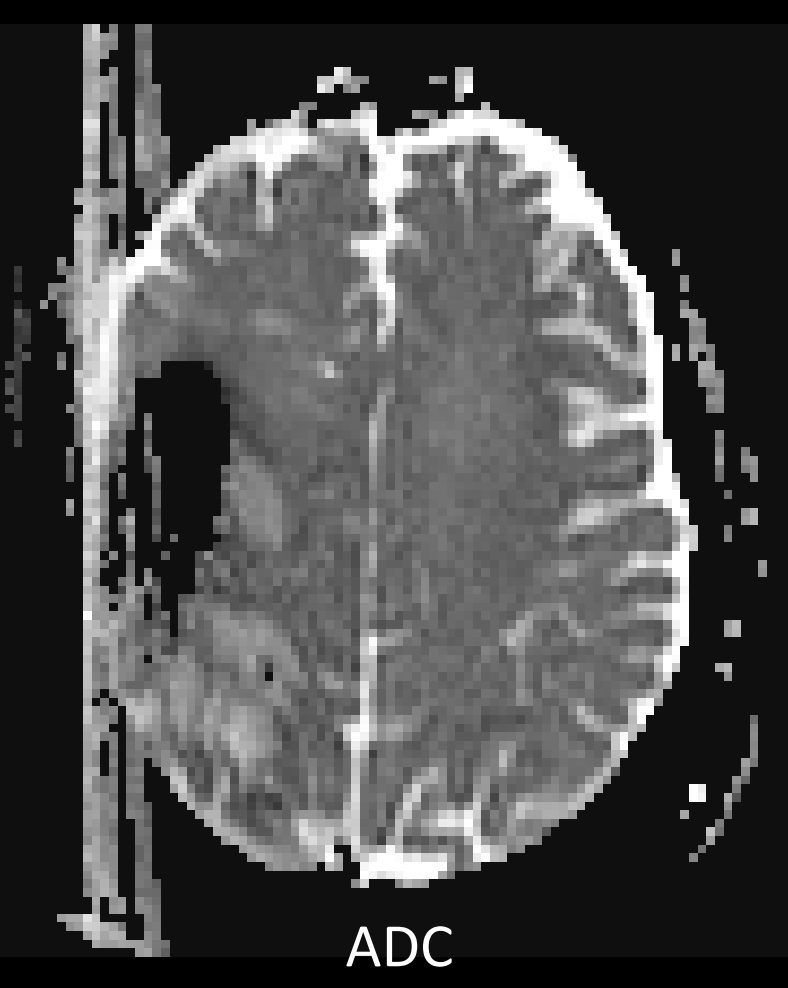

- Imaging findings: Multifocal punctate and ringenhancing enhancement with surrounding FLAIR signal and no associated restricted diffusion, predominantly in the right MCA distribution. Many of these foci are associated with susceptibility signal suggestive of hemorrhage. Right hemispheric pachymeningeal enhancement is also noted. Right ICA Pipeline stent is patent (not shown). 
Unexpected Post-Op Finding: Hydrophilic Gel Polymer Embolization

- Clinical course: Mild improvement in findings were seen following a short course of steroids.

- Follow up imaging: Repeat MRI 11 months after seizure onset showed complete resolution of previously seen findings.

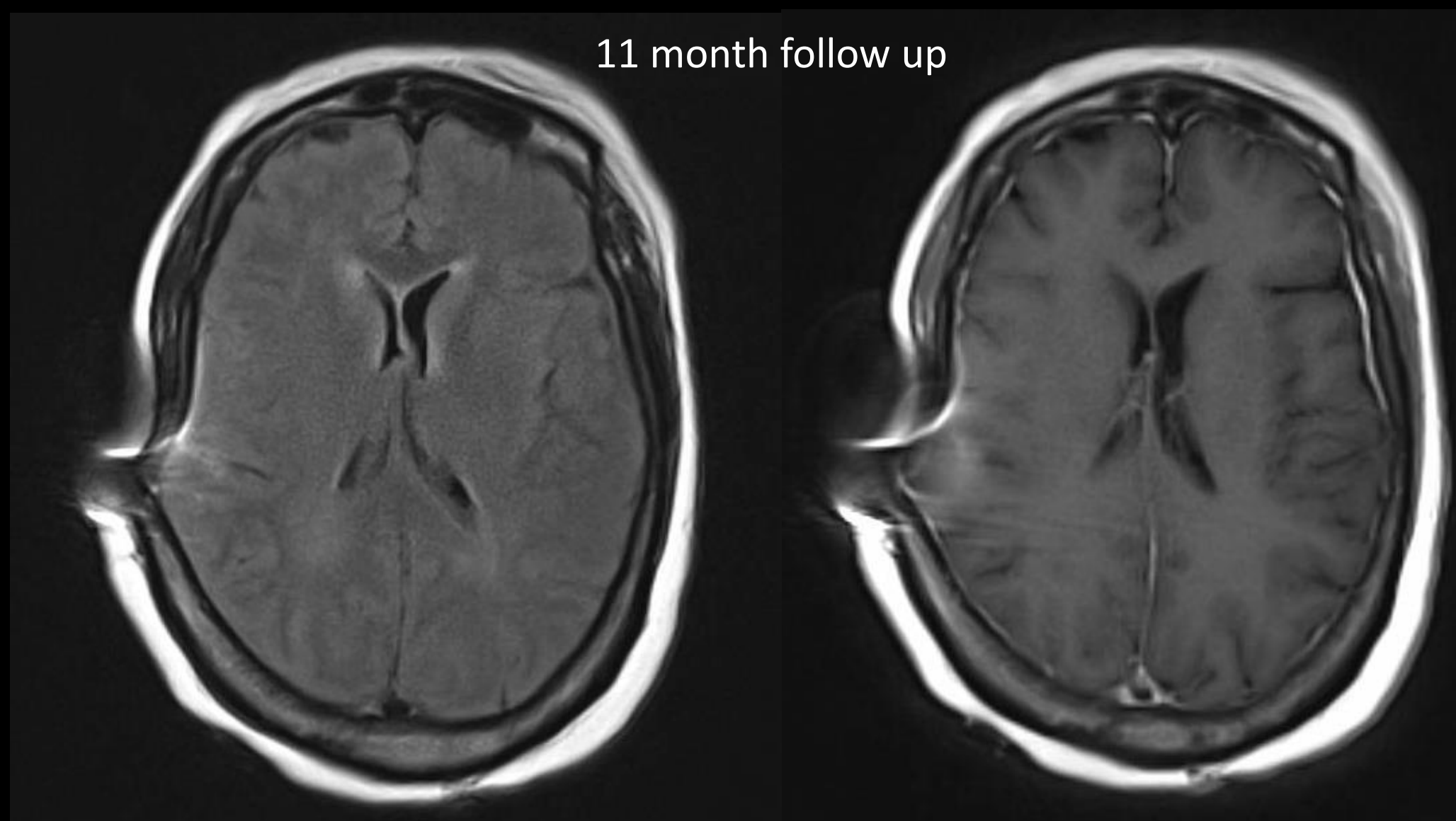

FLAIR
T1 Post-contrast

\section{TEACHING POINTS:}

1. Hydrophilic gel polymer embolization should be considered in patients with meningitis, multifocal infarct or enhancing lesions in a vascular distribution following any endovascular procedure that uses devices with hydrophilic polymers [2]

2. Polymer emboli can eventually dissolve, leading to resolution of surrounding granulomatous reaction 


\section{Infectious Complications}


Case 8 
- History: 45-year-old male transferred from outside hospital after developing worst headache of his life post coitus

- Pre-op Imaging: Diffuse subarachnoid hemorrhage centered at the anterior interhemispheric fissure, with intraventricular extension. Angiography revealed an irregular anterior communicating artery aneurysm most amenable to open clipping via frontal craniotomy.

- Although it is difficult to determine the location of a ruptured aneurysm by non-contrast $\mathrm{CT}$ alone, the distribution of subarachnoid blood products can be suggestive.

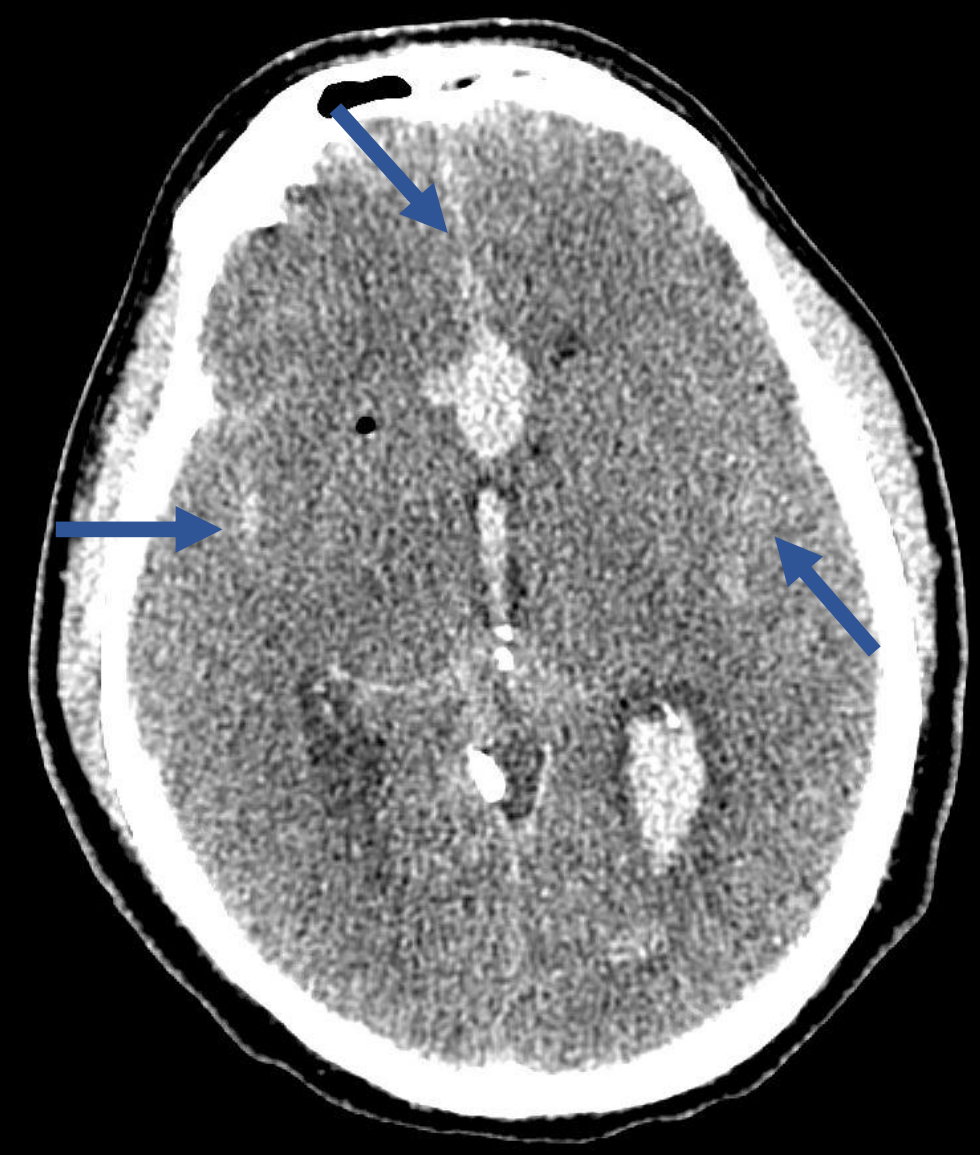

Non-contrast CT

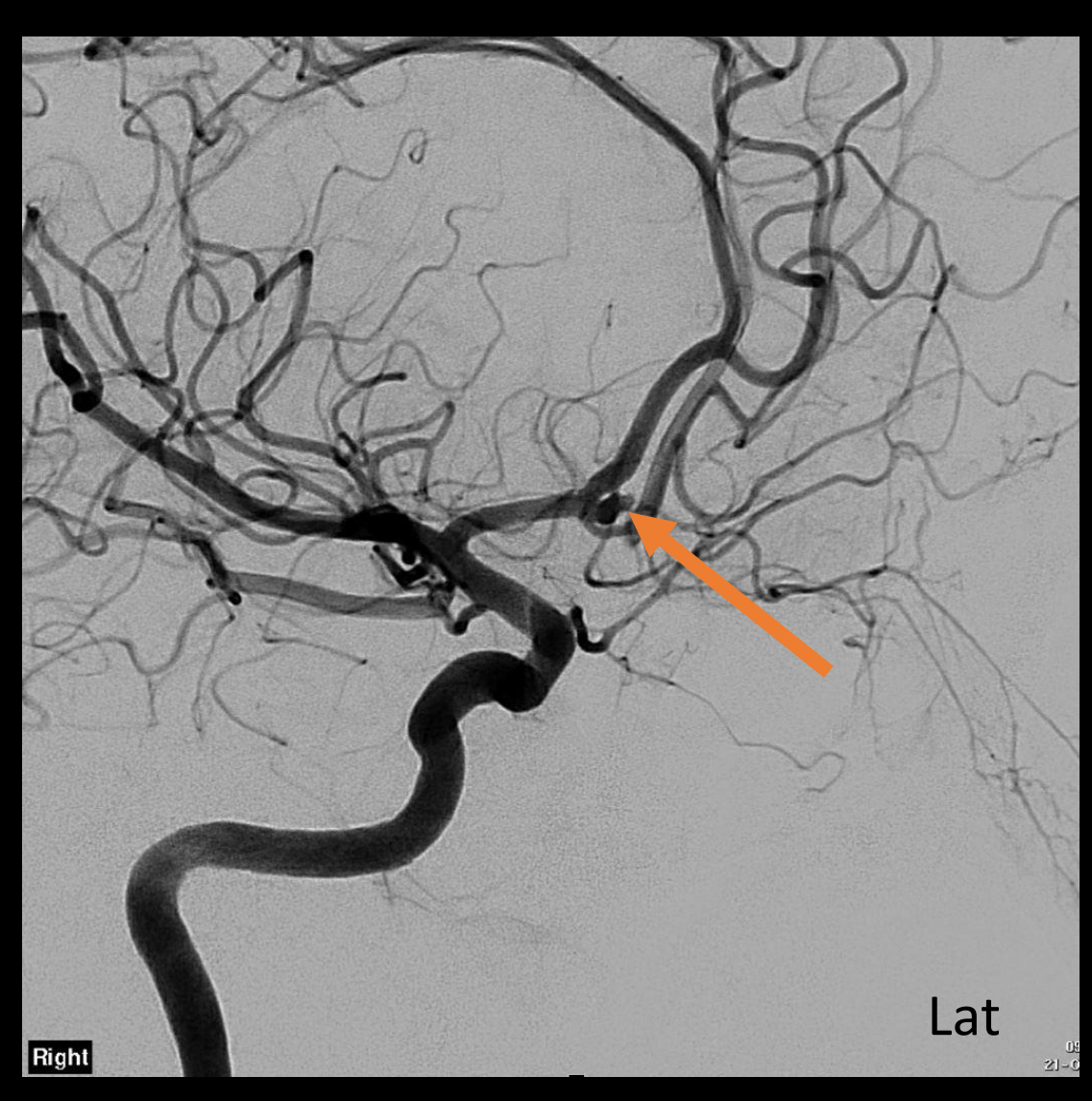

DSA, Right ICA injection

- Surgeries: 1) A-Comm aneurysm clipping via right pterional craniotomy

2) Decompressive suboccipital craniectomy for subsequent remote cerebellar intraparenchymal hemorrhage 


\section{Postoperative Unexpected Finding: Persistent fevers with Ventriculitis}

- Clinical course: Complicated post-op course with vasospasm, worsening hydrocephalus, difficulty weaning from the ventilator and inability tolerate EVD clamping. Continued clinical decline and fever prompted MRI 3 weeks post op

- Imaging: Periventricular enhancement and T2/FLAIR hyperintensity, with restricting intraventricular debris out of proportion to hemorrhage, consistent with ventriculitis. Bilateral ACA territory mass-like enhancement is related to evolving infarcts

- CSF grew Enterococcus Faecalis, likely nosocomial. Prolonged recovery requiring $P E G /$ Trach

TEACHING POINT: Persistent lack of clinical improvement post operatively and fevers suggests possible infectious complications and should prompt reimaging.
T1 Post-contrast

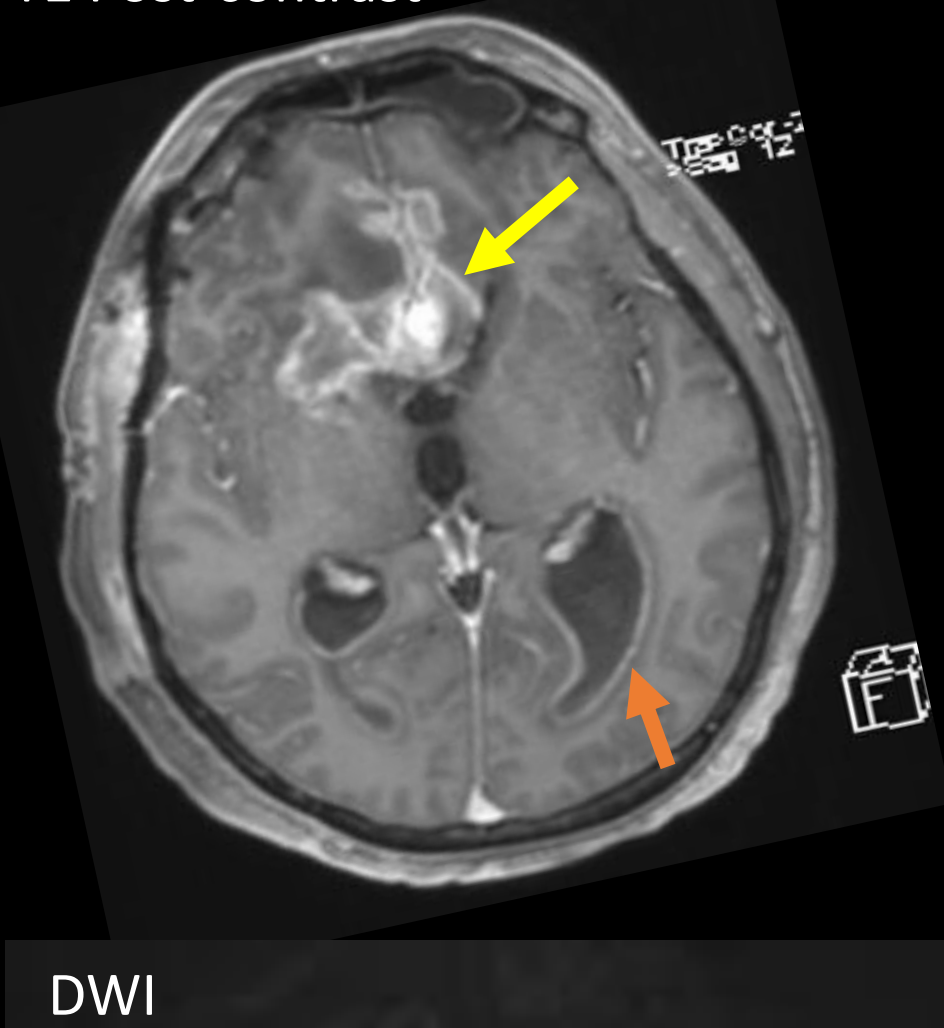

DWI

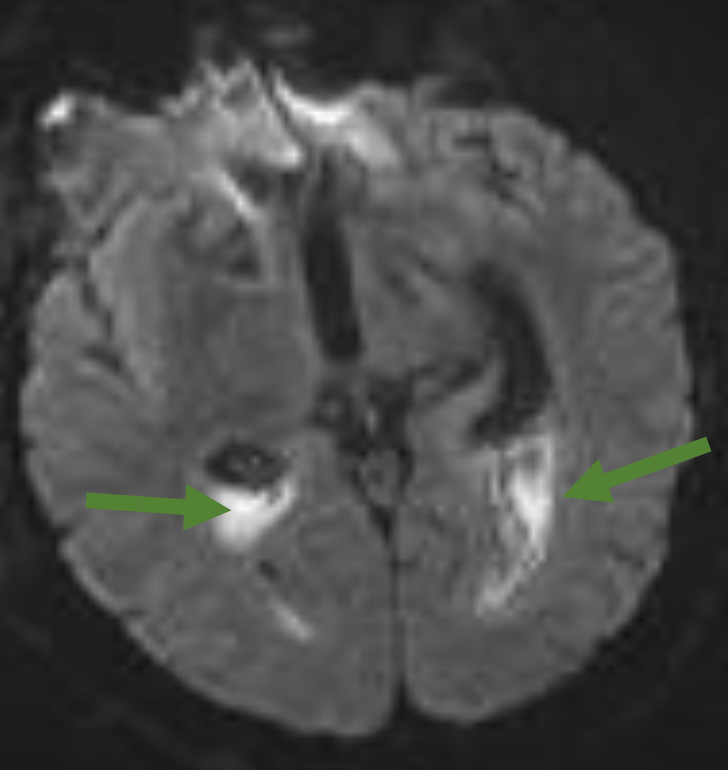

T2 FLAIR

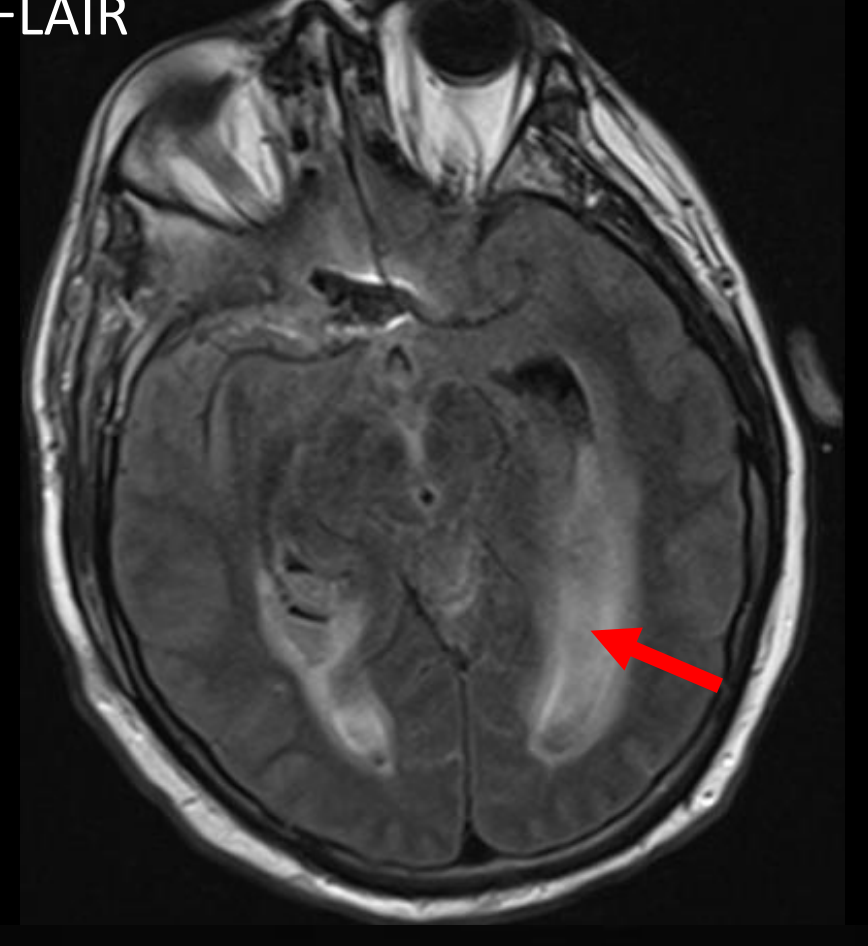

GRE

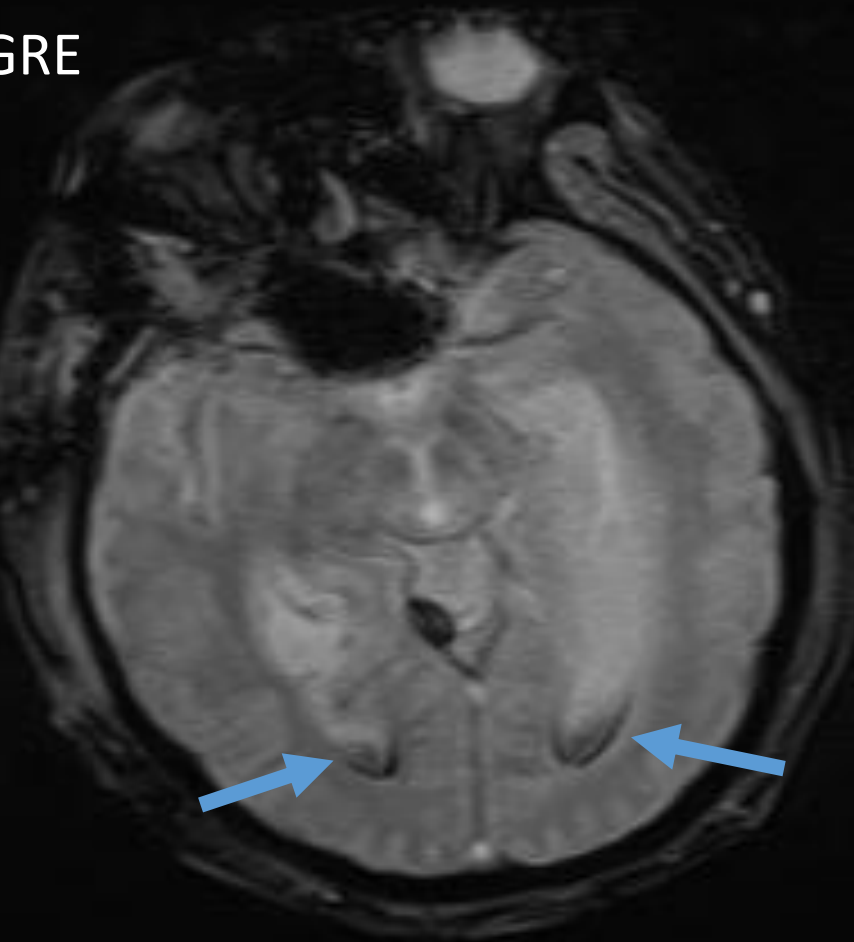


Case 9 

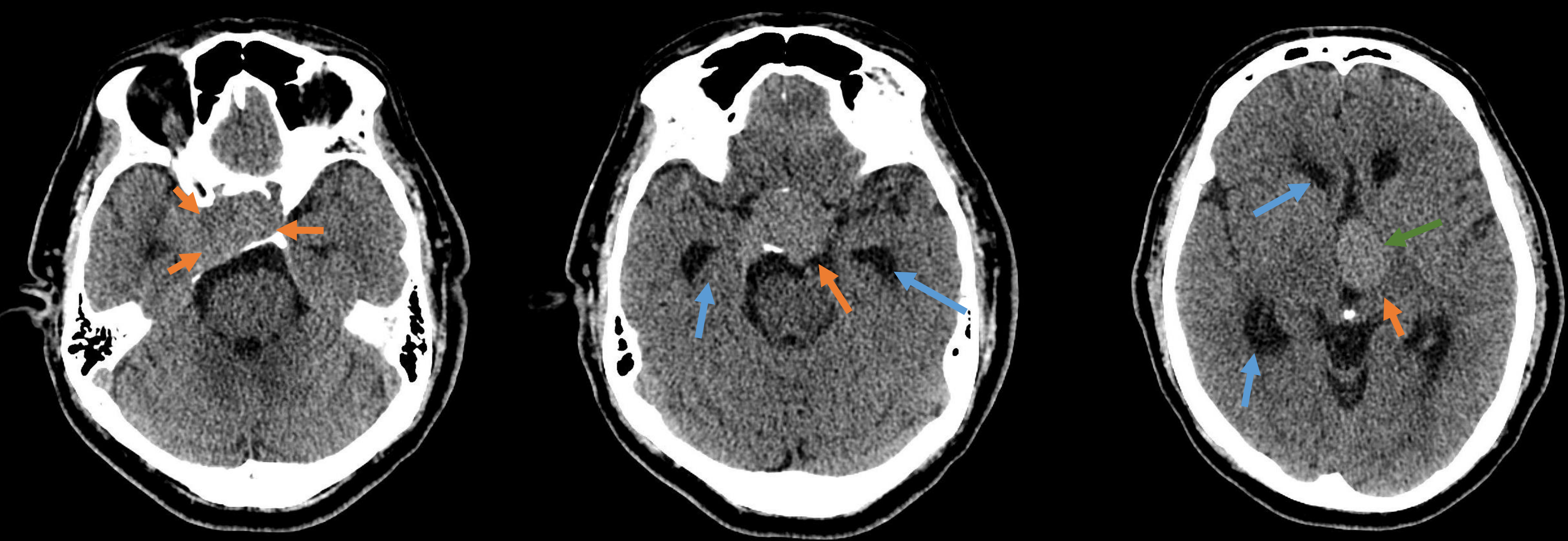

- History: 42-year-old male with progressive visual loss presented to the ER with acute worsening in vision

- Pre-op imaging: Non-contrast CT demonstrates a large sellar mass with suprasellar extension and mass effect on the anterior third ventricle and the foramen of Monroe, causing obstructive hydrocephalus.

- Surgery: Emergent transsphenoidal resection of a clinically non-functioning pituitary macroadenoma 


\section{CASE 9: Expected Immediate Post-Op Appearance Following Resection}

- Clinical Course: Given pre-op hydrocephalus, an external ventricular drain was placed prophylactically at the time of surgery. Post operatively patient returned to baseline visual fields.

- Post-op imaging: MRI demonstrates persistent hydrocephalus and trace pneumocephalus in the frontal horns, consistent with expected post-op appearance. Residual sellar mass is visualized.

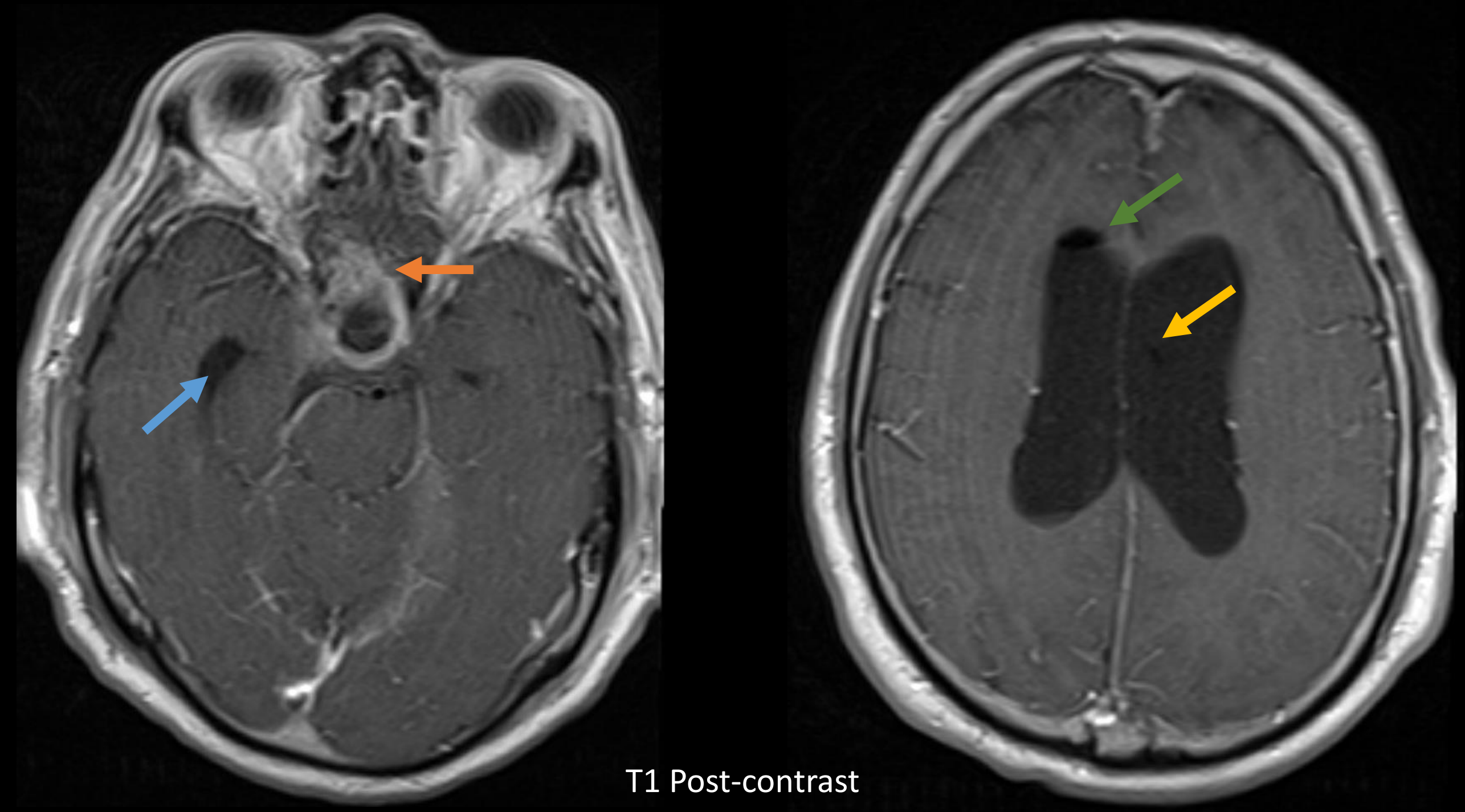




\section{Postoperative Unexpected Finding: Ventriculitis}

- Clinical Course: Patient experienced persistent fevers 2 weeks post op and did not tolerate EVD clamping

- Post-op Repeat Imaging: MRI demonstrated periventricular high T2 signal and enhancement with layering ventricular debris, new immediate post operative MRI, consistent with ventriculitis.

- CSF cultures collected 10 days post operatively grew K. Pneumonia and patient was treated with prolonged antibiotics

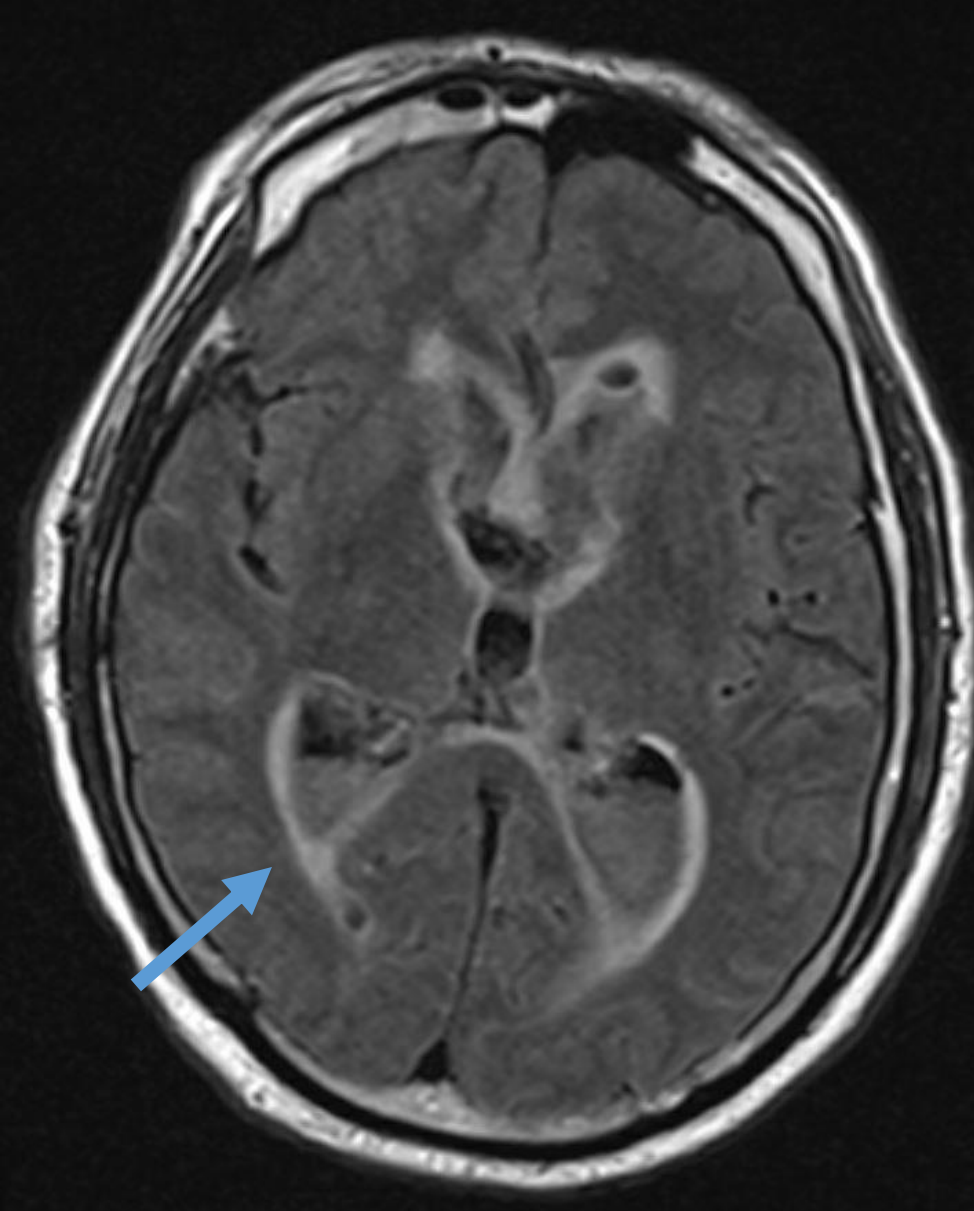

FLAIR

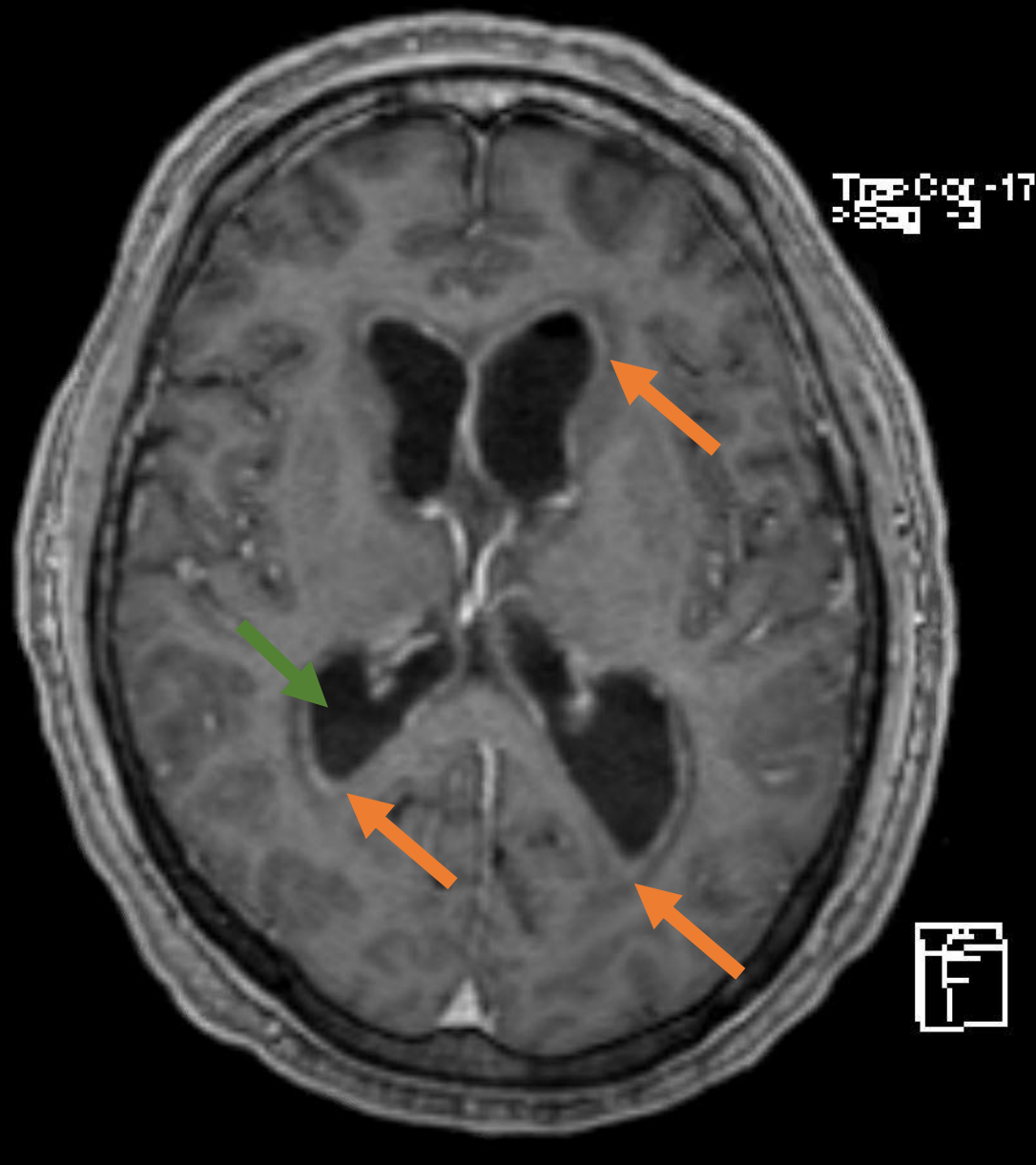

T1 post contrast

TEACHING POINT: Prolonged EVD as well as potential for CSF leak from transsphenoidal surgery present entry points for infections. Infection tracking throughout the CSF space can result in ventriculitis and/or meningoencephalitis 
Case 10 


\section{Case 10: Altered Mental Status in setting of CSF Leak After L3-4 laminectomy}

- History: 80-year-old male status post recent L3-4 laminectomy for neurological decompression, complicated by continuous CSF leak. Now presents with altered mental status/encephalopathy

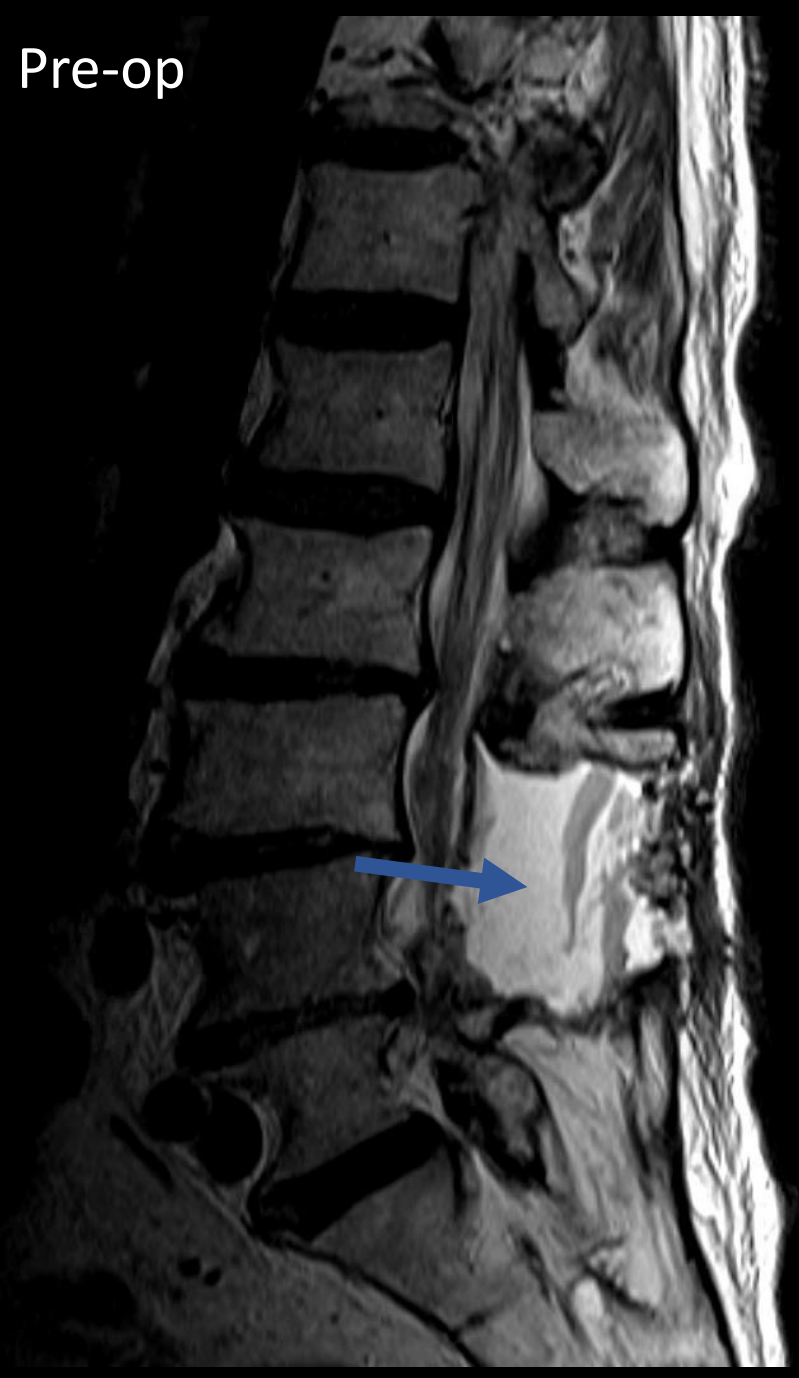

Sag T2

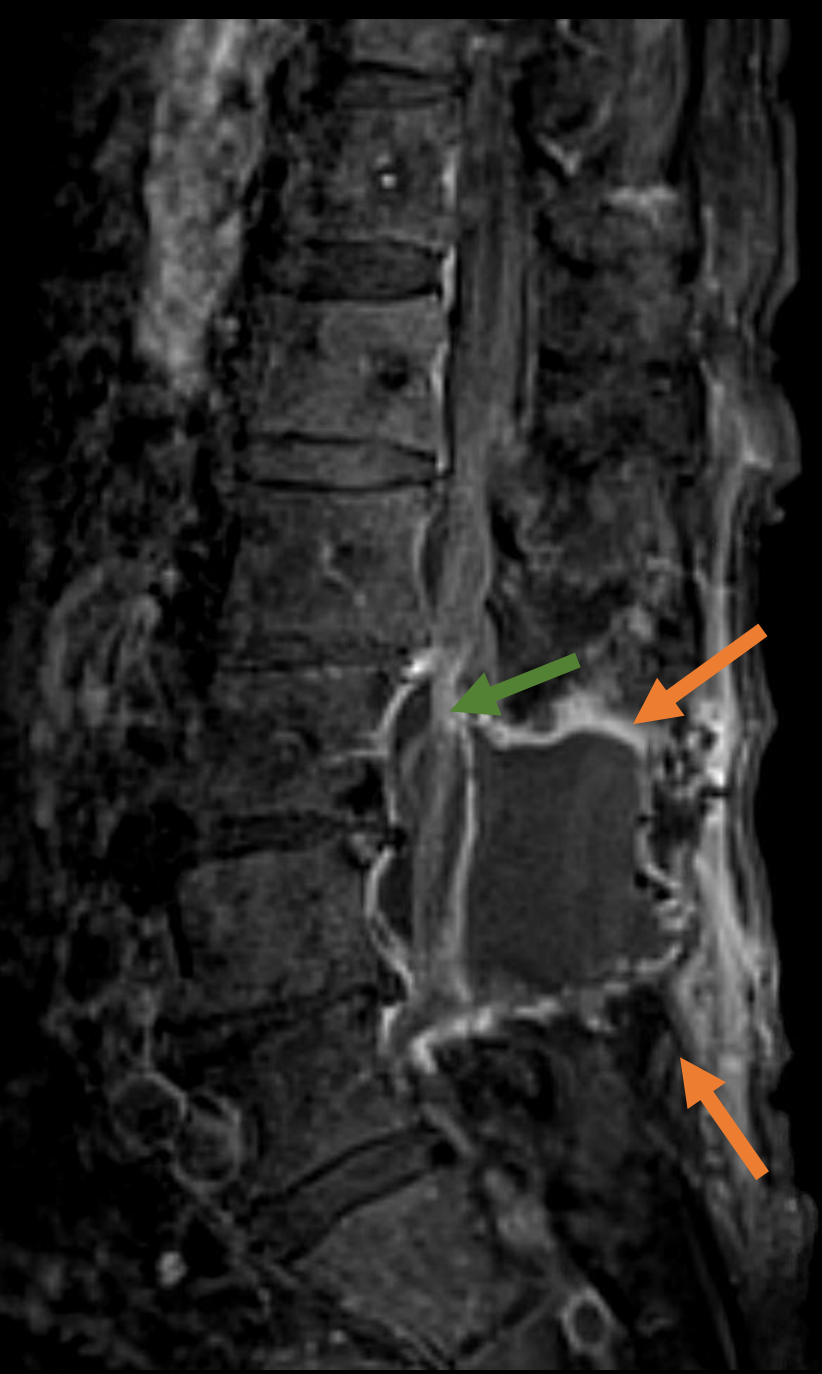

Sag T1 Fat Sat Post
- Pre-op Imaging: MRI demonstrated a large pseudomeningocele with peripheral enhancement. Enhancement of the nerve roots at the conus is suggestive of arachnoiditis

- Surgery: Pseudomeningocele washout and repair

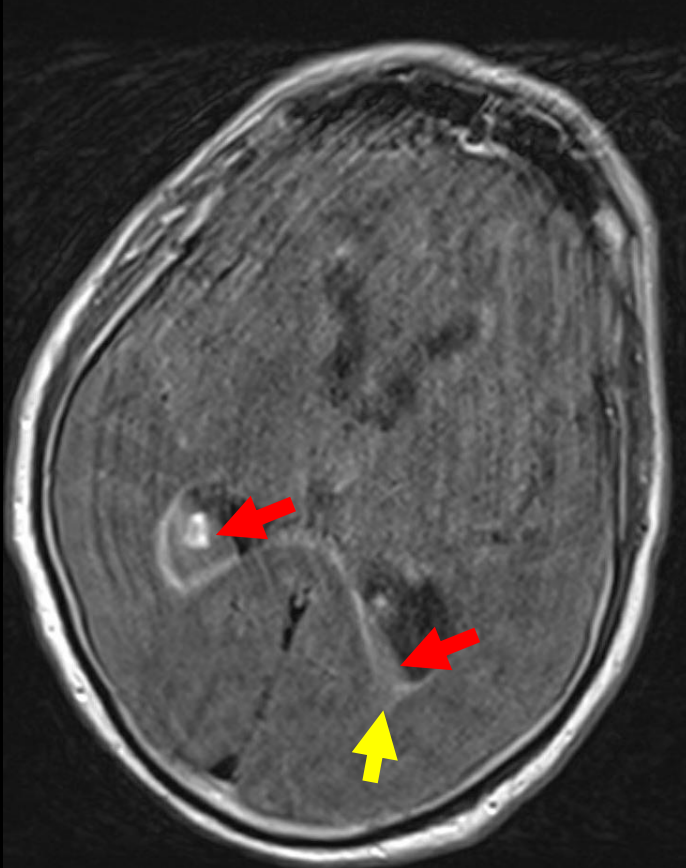

Post-op Imaging: Layered intraventicular debris with periventricular enhancement consistent with ventriculitis

TEACHING POINT: Persistent CSF leaks present an entry point for local infections including arachnoiditis, as well as ascending intracranial infections such as ventriculitis and/or meningoencephalitis 
Case 11 


\section{CASE 11: Occipital Metastases Resection}

- History: 75-year-old female with metastatic poorly differentiated pancreatic adenocarcinoma presents for follow up MRI after right occipital intraparenchymal hemorrhage 3 month prior

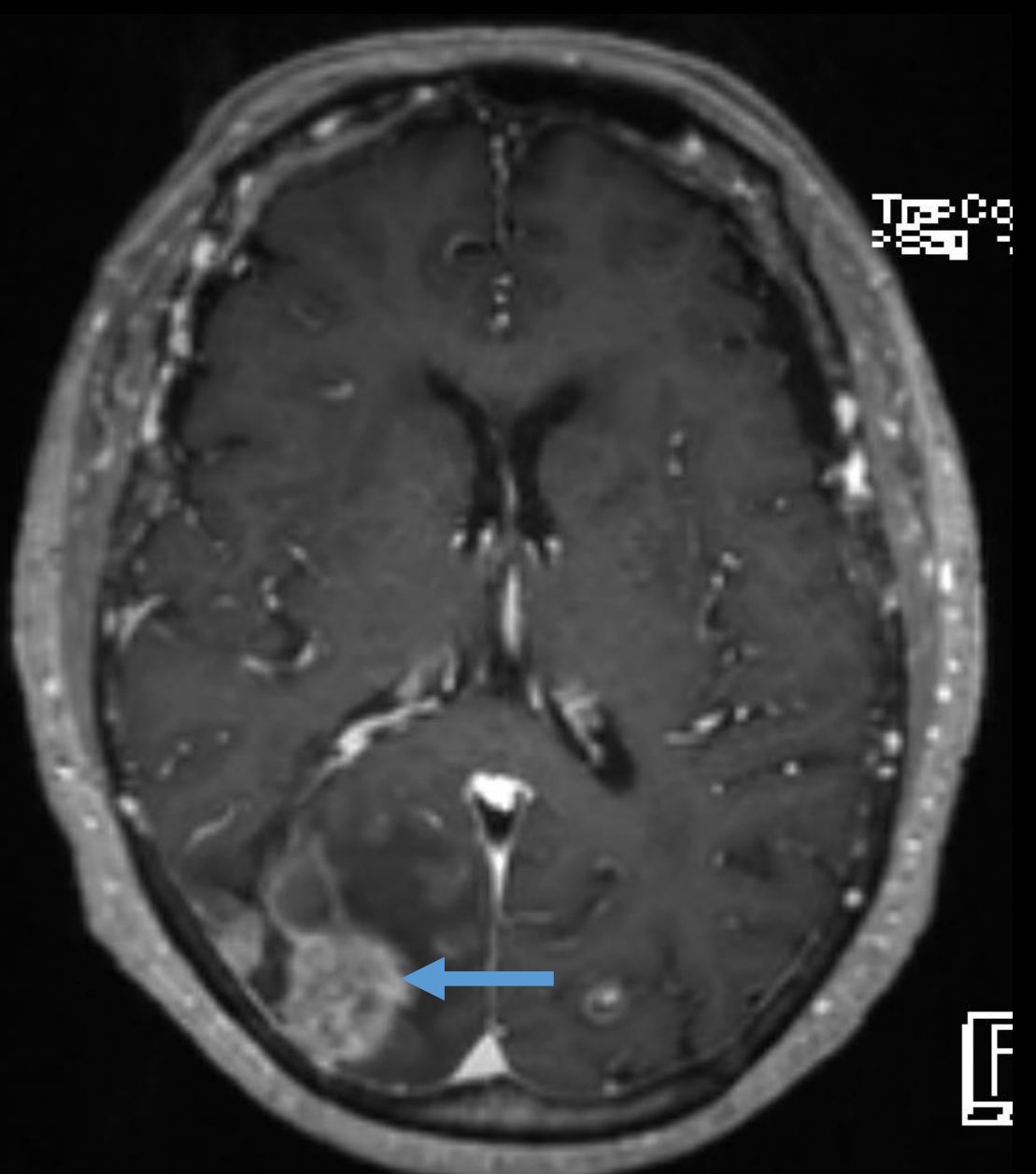

T1 Post

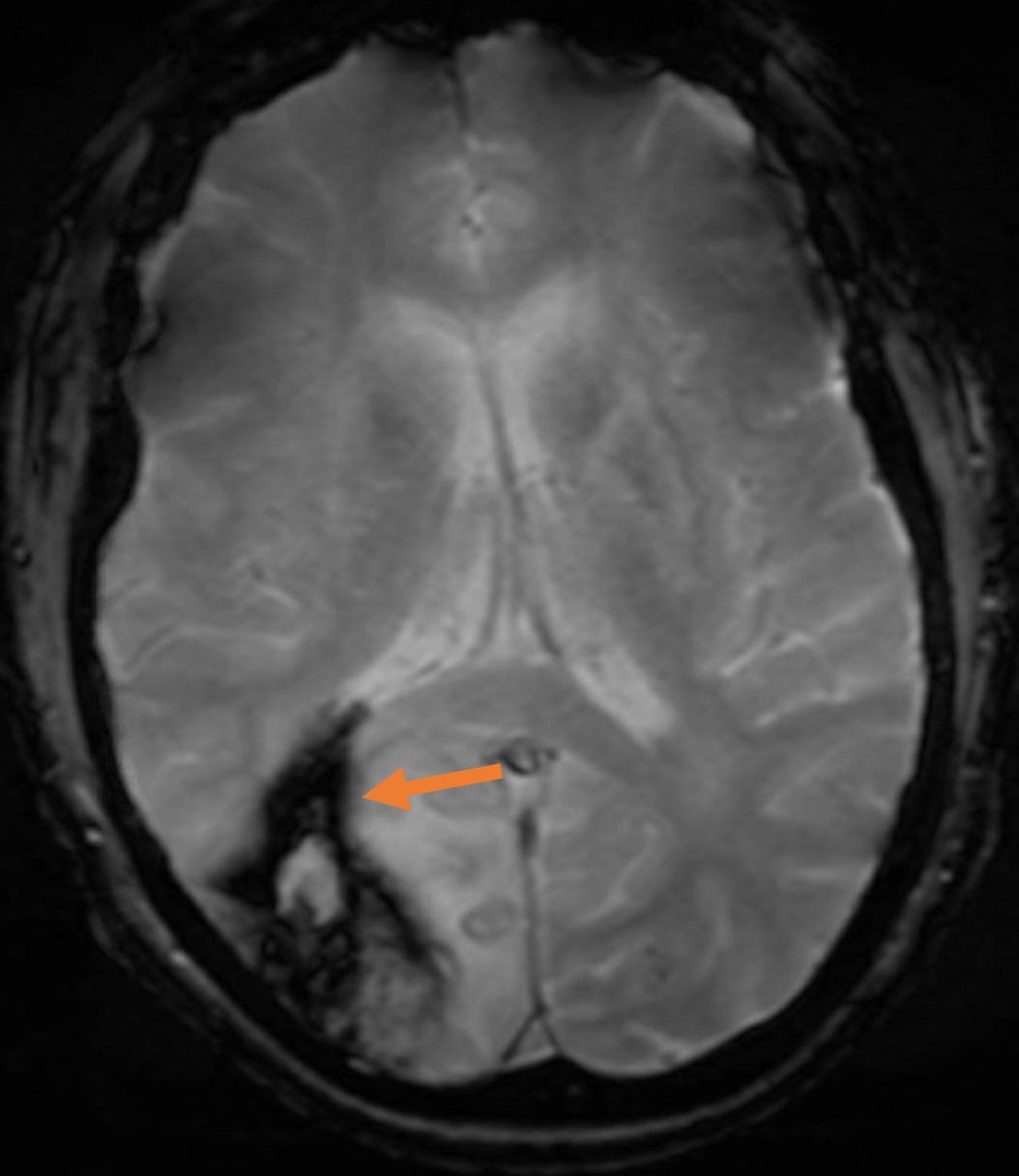

GRE
- Pre-op Imaging: Right parietooccipital enhancing mass with surrounding blood products and local mass effect

- Surgery: Mass resection and clot evacuation via right occipital craniotomy. Biopsy confirmed metastatic carcinoma.

- Clinical Course: Patient was discharged home after uneventful recovery 
- Clinical Course: Patient received radiation therapy following discharge. 1 month later patient developed increasing falls and gait disturbances. Of note, patient's uncontrolled diabetes compounded with Decadron resulted in difficult to control blood sugar
1 month follow-up
1 month follow-up MRI
Non-contrast CT
T1 Post
2-month follow-up MRI
T2 FLAIR
T1 Post
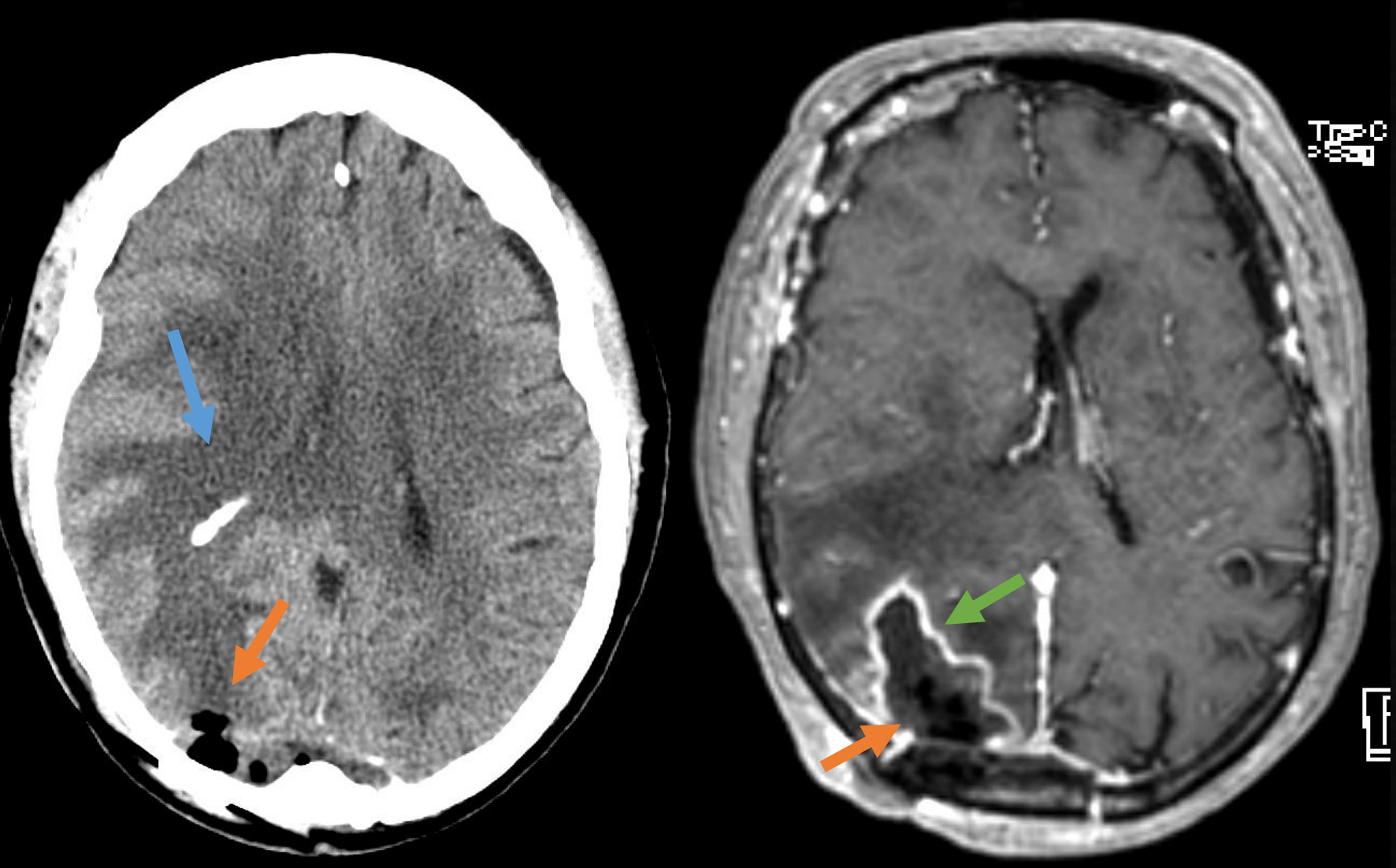

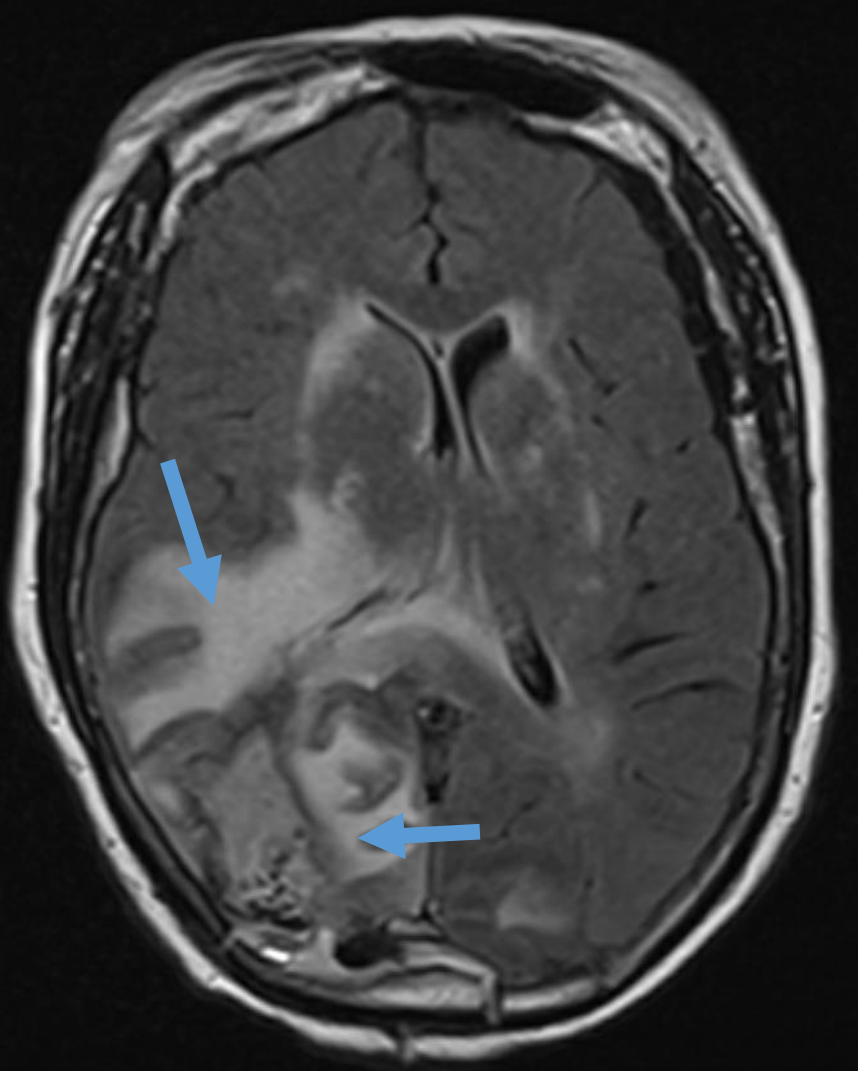

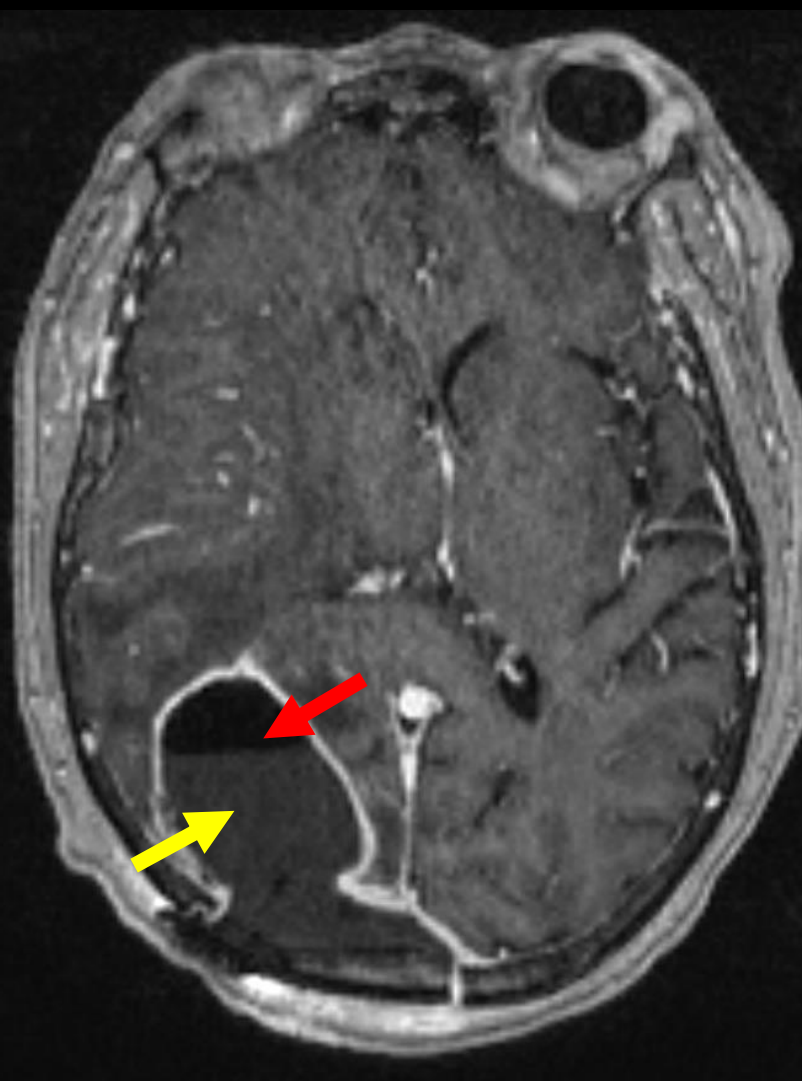

- Imaging: 1-month follow up CT and MRI demonstrated scattered pneumocephalus and fluid in the resection cavity with peripheral nodular enhancement. Significantly increased T2/FLAIR signal with new midline shift was attributed to postradiation changes. 2-month repeat MRI showed an enlarging rim-enhancing collection with air-fluid level, concerning for intracerebral abscess with gas-forming bacteria or skin communication 


\section{Postoperative Unexpected Finding: Recurrent Abscess and Empyema Following Washout}

- Surgery: Urgent washout and decompression with evacuation of frank purulent material

- Clinical Course: 2 weeks after abscess washout, patient developed worsening headache and confusion

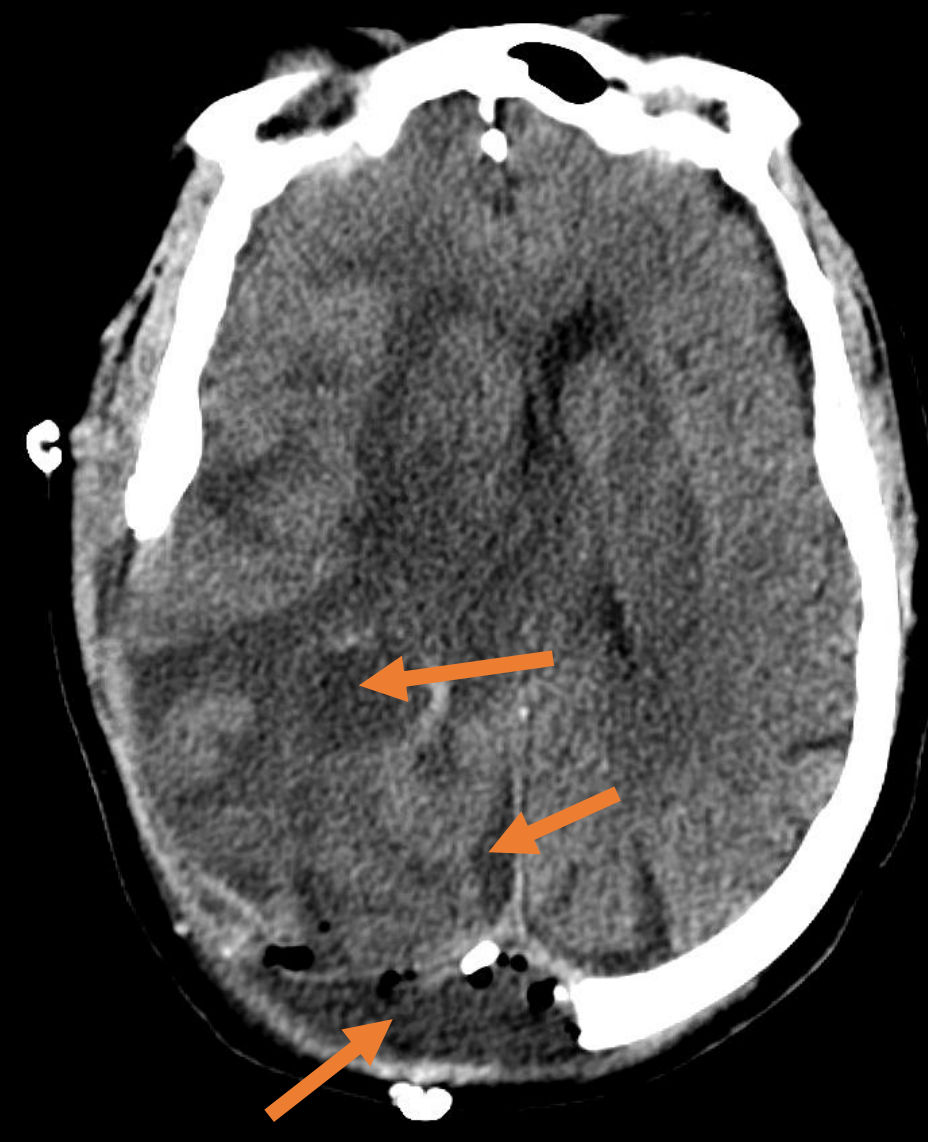

Non-contrast CT

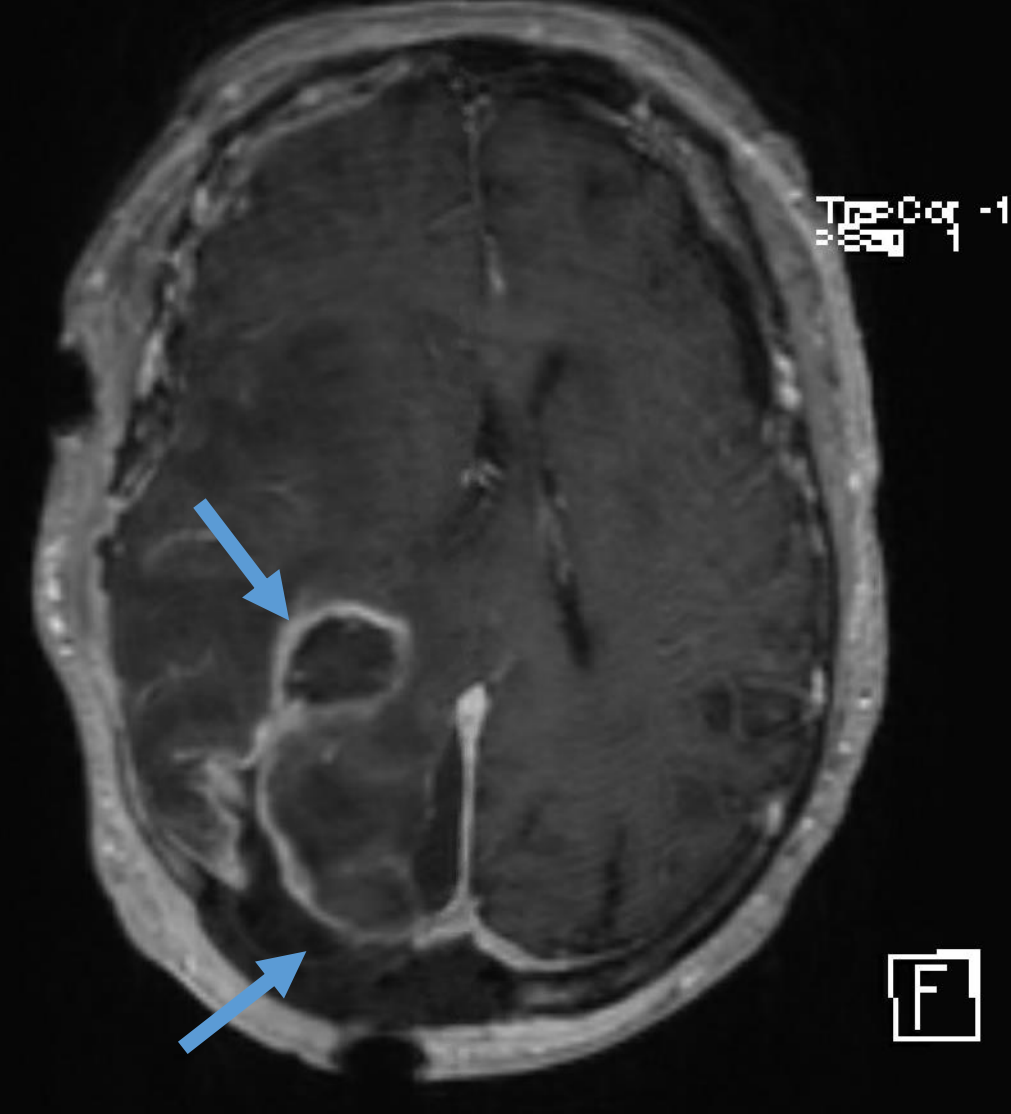

T1 Post-con

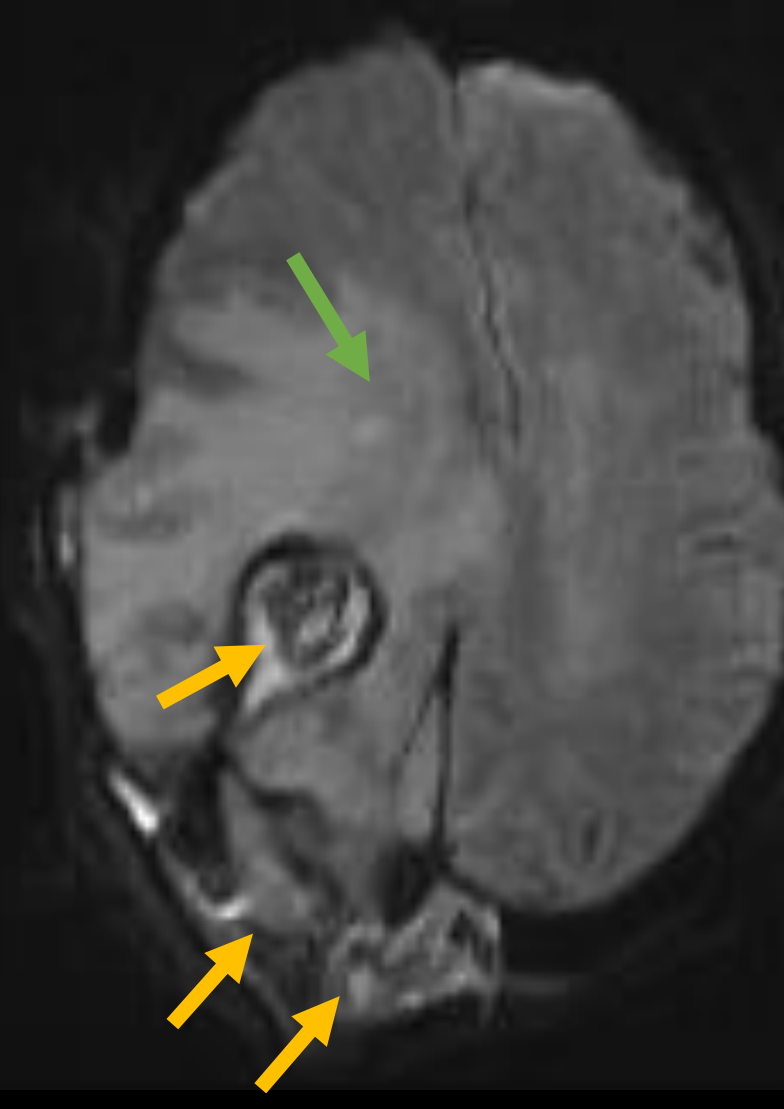

DWI

- Follow-Up Imaging: Persistent hypodense extra-axial and right parieto-occipital fluid collection with peripheral enhancement and internal diffusion restriction out of proportion to blood products suggestive of recurrent abscess/empyema. Redemonstrated vasogenic edema causing mass effect.

TEACHING POINT: Persistent/more than expected edema adjacent to the surgical bed over weeks to months raises suspicion for infectious process 


\section{TAKE-HOME POINTS}

- Post operative imaging is crucial to timely diagnosis of many common and uncommon presentations in the post surgical state

- Knowing the procedure performed and timeline of the patient's clinical course is crucial for correct diagnosis

- Persistent clinical signs and symptoms, failure to return to baseline post operatively and delayed presentations may suggest complications associated with the procedure which warrant specific treatment

- Reviewing the common and uncommon complications in the post operative period will aid the radiologist in making the timely diagnosis 